\title{
Chronic morphine-mediated upregulation of high mobility group box 1 in the spinal cord contributes to analgesic tolerance and hyperalgesia in rats
}

\author{
Junliang Qian ${ }^{1} \cdot$ Yanan Zhu ${ }^{1} \cdot$ Liying Bai ${ }^{3} \cdot$ Yan Gao $^{1,2}$ - Mingjun Jiang ${ }^{1} \cdot$ Fei Xing ${ }^{3}$ - Jian Zhang ${ }^{1}$. Wenchao Zhao ${ }^{1}$. \\ Hanwen $\mathrm{Gu}^{3} \cdot$ Yang $\mathrm{Mi}^{1} \cdot$ Yuan-Xiang $\mathrm{TaO}^{4} \cdot \mathrm{Ji}-\mathrm{Tian}_{\mathrm{Xu}}{ }^{1,2}$ (I)
}

Published online: 26 December 2019

(C) The American Society for Experimental NeuroTherapeutics, Inc. 2019

\begin{abstract}
Analgesic tolerance and hyperalgesia hinder the long-term utility of opioids. We examined whether spinal high mobility group box 1 (HMGB1) is involved in morphine tolerance and its underlying mechanisms by using a model of repeated intrathecal (i.t.) injections of morphine. The results showed that chronic i.t. morphine exposure led to increased expression of HMGB1, Toll-like receptor 4 (TLR4), and receptor for advanced glycation end products (RAGE) and their mRNAs in the dorsal horn. Morphine challenge also promoted HMGB1 expression and release in cultured spinal neurons, but these effects were inhibited by TAK-242, naloxone (antagonists of TLR4), and TLR4 siRNA. Intrathecal coadministration of morphine with TAK-242 or PDTC (inhibitor of NF- $K B$ activation) also reduced HMGB1 expression in the spinal cord. Repeated i.t. coinjections of morphine with glycyrrhizin (GL, an HMGB1 inhibitor) or HMGB1 siRNA prevented reduction of the maximal possible analgesic effect (MPAE) of morphine and alleviated morphine withdrawal-induced hyperalgesia. The established morphine tolerance and hyperalgesia were partially reversed when i.t. injections of GL or HMGB1 antibody started at day 7 of morphine injection. Repeated i.t. injections of morphine with HMGB1 siRNA inhibited the activation of NF- $\mathrm{kB}$, but not that of JNK and p38. A single i.t. injection of HMGB1 in naïve rats caused pain-related hypersensitivity and reduction in MPAE. Moreover, phosphorylated NF-KB p65, TNF- $\alpha$, and IL-1 $\beta$ levels in the dorsal horn were upregulated following this treatment, but this upregulation was prevented by coinjection with TAK-242. Together, these results suggest that morphine-mediated upregulation of spinal HMGB1 contributes to analgesic tolerance and hyperalgesia via activation of TLR4/NF-KB signaling, and the HMGB1 inhibitor might be a promising adjuvant to morphine in the treatment of intractable pain in the clinic.
\end{abstract}

Keywords High mobility group box $1 \cdot$ Morphine tolerance $\cdot$ Hyperalgesia $\cdot$ Signal pathway $\cdot$ Spinal cord

\section{Introduction}

Chronic pain has become a major public health problem. Although recent advances have been made in the

Junliang Qian, Yanan Zhu and Liying Bai contributed equally to this work.

Ji-Tian Xu jtxu@zzu.edu.cn

1 Department of Physiology and Neurobiology, Zhengzhou University School of Basic Medical Sciences, 100 Science Avenue, Zhengzhou 450001, China

2 Neuroscience Research Institute, Zhengzhou University Academy of Medical Sciences, 100 Science Avenue, Zhengzhou 450001, China therapeutic management of chronic pain, morphine and related $\mu$-opioid receptor agonists are still the gold standard for its pharmacological treatment in clinical settings. However, opioid treatments often result in several

3 Department of Anesthesiology, The First Affiliated Hospital, Zhengzhou University, 40 Daxue Road, Zhengzhou 450052, China

4 Department of Anesthesiology, New Jersey Medical School, Rutgers, The State University of New Jersey, 185 S. Orange Ave., MSB, E-661, Newark, NJ 07103, USA 
adverse side effects, among which opioid-induced analgesic tolerance and hyperalgesia largely hinder opioids' long-term utility $[1,2]$. Although considerable efforts have been expended on developing an understanding of the molecular and cellular processes of opioidinduced tolerance and hyperalgesia, these conditions are still ineffectively managed by existing drugs [3-5]. Therefore, the identification of novel molecular machinery involved in opioid-induced tolerance is still a major concern in the field of pain medicine research [6].

High mobility group box 1 (HMGB1), a nonhistone nuclear protein, is well known to bind to DNA and stabilize DNA interactions with transcription factors to regulate gene transcription [7]. Emerging evidence has recently shown that HMGB1 is a potent proinflammatory mediator when extracellularly present in various organs $[8,9]$. The basic pathway of HMGB1 release from cells is active secretion, which is triggered by cellular signal transduction through the interaction of plasma membrane receptors with extracellular products $[10,11]$. Compelling evidence shows that the active release of HMGB1 is modulated by Toll-like receptor 4 (TLR4) signaling and inflammatory mediators [9, 12]. The long-term use of opioids, such as morphine, not only creates analgesia via classical opioid receptors $(\mu, \delta$, and $\kappa)$, but also results in sterile neuroinflammation in the central nervous system via TLR4 signaling activation [13-15]. Therefore, we postulate that chronic morphine exposure may affect the active release of HMGB1 in the nervous system.

It has been well documented that chronic morphine exposure-induced spinal neuroinflammation plays critical role in the development and maintenance of morphine analgesic tolerance and hyperalgesia $[16,17]$. Therefore, we promised that as pro-inflammatory cytokines and chemokines, spinal HMGB1 might be another important morphine-induced inflammatory mediator and contribute to the development of morphine tolerance. However, the expression and the role of HMGB1 in morphine analgesic tolerance and morphine withdrawal-induced hyperalgesia still remain elusive. The results of present study have provided evidence, for the first time in our knowledge, that inhibition of spinal HMGB1 activity effectively prevented the reduction of analgesic tolerance, and alleviated morphine withdrawal-induced hyperalgesia.

\section{Materials and Methods}

\section{Animal Preparation}

Male Sprague-Dawley rats weighing 200-300 g and male C57BL/6 mice (7-8 weeks of age, weighing 18-
$21 \mathrm{~g})$ were used and randomly divided into different groups. The female rats were also used in other groups to evaluate the sex variable in the current study. The animals were purchased from the Laboratory Animal Center of Zhengzhou University, Zhengzhou, China, and housed in separate cages with free access to food and water. The room temperature was maintained at 23 $\pm 2^{\circ} \mathrm{C}$ under a 12:12-hour light-dark cycle. All animal experimental procedures were approved by the Institutional Animal Care and Use Committee of Zhengzhou University in China and were carried out in accordance with the National Institutes of Health's guidelines on animal care and the ethical guidelines for the experimental investigation of pain in conscious animals.

\section{Intrathecal Catheterization and Drug Delivery}

Drugs were delivered intrathecally. Intrathecal catheterization was performed following the procedures described by Storkson et al [18] with modifications. In brief, a polyethylene-10 (OD, $0.61 \mathrm{~mm}$; ID, $0.28 \mathrm{~mm}$ ) catheter was inserted into the rat's subarachnoid space through the intervertebral space between L5 and L6, and the tip of the catheter was placed at the L5 level of the spinal cord. The rats were allowed to recover for 7 days before behavioral testing or i.t. injections of the drugs. The position of the catheter was confirmed postmortem. Animals that displayed any abnormal neurological signs were excluded from the experiments. Morphine hydrochloride was purchased from the Northeastern Pharmaceutical Group (Shenyang, China) and diluted in normal saline prior to use. The HMGB1 inhibitor glycyrrhizin (GL), the Tolllike receptor4 (TLR4) antagonist TAK-242, naloxone, and the NF- $\mathrm{KB}$ activation inhibitor pyrrolidinedithiocarbamate (PDTC) were purchased from Sigma (Sigma, St. Louis, USA) and freshly dissolved daily in normal sterile saline containing 10\% DMSO prior to use. Anti-HMGB1 neutralizing antibodies were purchased from CST (Cell Signaling Technology, USA) and diluted with sterile artificial cerebrospinal fluid (ACSF) containing $126.6 \mathrm{mM} \mathrm{NaCl}$, $2.5 \mathrm{mM} \mathrm{KCl}, 2.0 \mathrm{mM} \mathrm{MgCl} 2$, and $1.3 \mathrm{mM} \mathrm{CaCl}_{2}$. The recombinant disulfide HMGB1 was purchased from HMGBiotech (HMGBiotech S.r.l., Milano, Italy), and the dose used for i.t. injections $(2 \mu \mathrm{g} / 10 \mu \mathrm{l})$ was based on that used in a previously published paper [19]. The intrathecal (i.t.) doses of GL $(10,20,40 \mu \mathrm{g} / 10 \mu \mathrm{l})[20,21]$, TAK-242 $(20 \mu \mathrm{g} / 10 \mu \mathrm{l})$ [22], PDTC $(0.5 \mu \mathrm{g} / 10 \mu \mathrm{l})$ [23], and the antiHMGB1 antibody $(2 \mu \mathrm{g} / 10 \mu \mathrm{l})$ [24] used in the current experiment were based on those used in previous studies. The doses of TAK-242 $(2 \mu \mathrm{M})$ [25], CTOP $(10 \mu \mathrm{M})$, and naloxone $(100 \mu \mathrm{M})$ [26] used in the current in vitro study were also based on previous published papers. 
Induction of Morphine Tolerance and Behavioral Tests for Nociceptive

Morphine tolerance was induced following the methods described by Lim [27] and our previously described method [26]. Briefly, rats were injected intrathecally with morphine $(10 \mu \mathrm{g} / 10 \mu \mathrm{l})$ twice daily for 6 consecutive days. Rats receiving i.t. saline (vehicle) $(10 \mu$ l twice daily) for 6 days served as the control group. All rats were acclimated to the testing environment for at least 3 days prior to baseline measurements, and the pain-related behavioral testing was carried out in a blinded manner. At least three persons were involved in each experiment and only one knew the design of the study for each test. The tail-flick test was performed with baseline latencies of 4-5 $\mathrm{s}$ and a cutoff time of $10 \mathrm{~s}$ using an analgesic meter (Model 33B Tail Flick Analgesia Meter, IITC Life Science, Woodland Hills, CA, USA). At least three trials were performed for each rat with an interval of 1 min between the tests and with a change in the tail area receiving radiant heat stimulation at each trial. The percentage of morphine's maximal possible analgesic effect (\% MPAE) was determined by comparing the baseline tail-flick latencies $(\mathrm{BL})$ and the latencies after drug injection (TL) using the following equation: \% MPAE $=[(\mathrm{TL}-\mathrm{BL}) /(10-\mathrm{BL})] \times 100 \%$ (the constant 10 refers to the cutoff time in seconds). Baseline tail-flick latencies were measured 1 day before drug injection, and 0.5 hour after intrathecal morphine or vehicle injection on the mornings of days $1,3,5,7,9$, or 12 to assess the development and maintenance of morphine tolerance.

To assess mechanical sensitivity, the paw withdrawal threshold (PWT) was determined by applying mechanical stimuli to the plantar surface of the hind paw using von Frey hairs $(0.4,0.6,1,1.4,2,4,6,8$, and $15 \mathrm{~g})$, and the $50 \%$ PWT was determined using the up-down method [28]. Heat hypersensitivity was determined by measuring the paw withdrawal latency (PWL) using the plantar test (7370, Ugo Basile, Comeria, Italy) according to the method described by Hargreaves et al [29]. Baseline PWT and PWL values were measured 1 day before drug injection, and 1 day after morphine withdrawal to assess levels of hyperalgesia.

Spontaneous pain was detected by the single trial conditioned place preference (CPP) following the method described previously [30]. Preconditioning to an automated 3 chambers CPP box was performed, started at day 7 of morphine i.t. injection. All rats underwent a 2-day habituation period in which they were exposed to the environment with full access to all chambers for 30 min. Behavior was recorded for $15 \mathrm{~min}$ on day 3 and analyzed to verify no preconditioning chamber preference. The following day (one day after morphine withdrawal), rats received the appropriate control (i.t. vehicle) paired with a randomly chosen chamber in the morning, and the appropriate drug treatment (Group 1: rats received vehicle i.t. injection served as control; Group 2: rats received clonidine $(10 \mu \mathrm{g})$ i.t. injection served as positive control; Group 3: rats received GL $(40 \mu \mathrm{g})$ paired with the other chamber 4 hours later in the afternoon. Chamber pairings were counterbalanced. On test day, 20 hours following the afternoon pairing, rats were placed in the CPP box with access to all chambers and their behavior were recorded for $15 \mathrm{~min}$ for analysis of chamber preference.

\section{Western Blotting}

Western blotting was performed according to our previous published procedures $[26,31]$. Briefly, the animals were sacrificed by decapitation at a designated time point. The spinal dorsal horn of the lumbar enlargement was harvested and placed temporarily in liquid nitrogen. Then, the samples were homogenized with ice-cold lysis buffer (10 mM Tris, $5 \mathrm{mM}$ EGTA, $0.5 \%$ Triton X-100, $2 \mathrm{mM}$ benzamidine, $0.1 \mathrm{mM}$ PMSF, $40 \mathrm{mM}$ leupeptin, $150 \mathrm{mM} \mathrm{NaCl}$, and $1 \%$ phosphatase inhibitor cocktail 2 and 3). The crude homogenate was centrifuged at $4^{\circ} \mathrm{C}$ for $15 \mathrm{~min}$ at $3000 \mathrm{r} / \mathrm{min}$, and the supernatants were collected. Then, $50 \%$ of the supernatant was removed and considered to be the total soluble fraction. The remaining supernatant was centrifuged at $37,000 \mathrm{~g}$ for 1 hour at $4^{\circ} \mathrm{C}$. The supernatant was considered to be the crude cytoplasmic fraction, and the pellet was considered to be the crude plasma membrane fraction. After the protein concentrations were measured, the samples were heated for $5 \mathrm{~min}$ at $99^{\circ} \mathrm{C}$, and $30-60 \mu \mathrm{g}$ protein was loaded onto $10-12.5 \%$ SDSpolyacrylamide gels. The proteins were electrophoretically transferred onto PVDF membranes. The blotting membranes were blocked with $3 \%$ nonfat dry milk for 1 hour and incubated overnight at $4{ }^{\circ} \mathrm{C}$ with a primary antibody. The following primary antibodies were used: rabbit anti-HMGB1 (1:1000; Sigma, St. Louis, USA), rabbit anti-TLR4 (1:1000; Abcam, Cambridge, MA), rabbit anti-RAGE (1:1000; Abcam), rabbit anti-NF-kB p-p65 (1:1000; Abcam), mouse anti-IkB- $\alpha$ (1:300; Senta Cruz Biotech), rabbit anti-IL-1 $\beta$ (1:300; Abcam), rabbit anti-TNF- $\alpha$ (1:300; Abcam), rabbit anti-pp38 (1:500; Cell Signaling Technology, USA), rabbit anti-pJNK (1:500; Cell Signaling), and mouse anti- $\beta$-actin (1:10,000; Sigma). The proteins were detected with horseradish peroxidase-conjugated anti-mouse or anti-rabbit secondary antibodies (1:3000; Bio-Rad, Philadelphia, PA), visualized using enhanced chemiluminescence reagents (Bio-Rad) and detected by a ProteinSimple imager (FluorChem E, USA). The intensities of the blots were quantified using a computerassisted imaging analysis system (ImageJ; NIH, USA). Then, the ratios of protein expression to $\beta$-actin expression were 
calculated for each group, and each value of the control was normalized to the mean of the control group. Next, the ratio of each group (including that of the control group) was divided by that of the control group.

\section{Immunohistochemistry}

Immunohistochemistry was carried out following the procedures described by Ji et al [32] and our previous published procedures [33]. Briefly, after 5 or 7 days of i.t. injections of morphine, rats were deeply anesthetized with isoflurane and perfused through the ascending aorta with normal saline, followed by $4 \%$ paraformaldehyde in $0.1 \mathrm{~mol} / \mathrm{L}$ phosphate buffer. After perfusion, the lumbar (L4-6) spinal cord was removed and postfixed in the same fixative for $3 \mathrm{~h}$, and replaced in 30\% sucrose in PBS for two nights. Transverse spinal sections $(25 \mu \mathrm{m})$ were cut on a cryostat (Leica, CM1950) and prepared for immunofluorescence staining. Sections were randomly selected and placed in different wells of a 24-well plate. After washing with PBS, the sections were blocked with 5\% goat serum in $0.3 \%$ Triton X-100 for 1 hour at $37^{\circ} \mathrm{C}$, and incubated with primary antibody overnight at $4^{\circ} \mathrm{C}$. For double immunofluorescence staining, the sections were incubated in a mixture of primary antibody with a specific spinal cell marker antibody overnight and then incubated with a mixture of $\mathrm{Cy} 3$ - and FITC-conjugated secondary antibodies for 1 hour at $37^{\circ} \mathrm{C}$. The stained sections were mounted on slides and examined under a fluorescence microscope (Olympus BX53, Tokyo, Japan) or high-resolution laser confocal fluorescence microscope (Nikon A1R MP+, Japan) and images were captured with a CCD spot camera. The primary antibodies were rabbit anti-HMGB1 (1:200; Sigma), rabbit anti-TLR4 (1:200; Abcam), and rabbit anti-RAGE (1:200; Abcam). The spinal cell-specific marker antibodies were monoclonal neuron-specific nuclear protein (NeuN) (neuronal marker, 1:200; Chemicon, Billerica, MA), anti-microtubuleassociated protein 2 (MAP2) (1:200; Alexa Fluor ${ }^{\circledR} 488$ Conjugate Antibody, Millipore), glial fibrillary acidic protein (GFAP) (astrocyte marker, 1:400; Chemicon), and OX42 (CD11b, microglia marker, 1:200; Chemicon).

The specificity of anti-HMGB1 antibody was evaluated by preincubating the antibody with its specific antigen, HMGB1, as described previously [34]. The specificity of the TLR4 antibody was examined by i.t. injection of TLR4 siRNA. The results of the immunofluorescence staining showed a clear decrease in TLR4-immunoreactivity in spinal cord sections after rats received TLR4 siRNA treatment (data not shown). The specificity of the RAGE antibody was evaluated by omitting the primary antibody during immunofluorescence staining, and further examined by Western blot, in which the rabbit anti-RAGE antibody detected a band at a size that correlated with the molecular weight of RAGE.

\section{RNA Extraction and Real-time Quantitative RT-PCR}

Real-time quantitative RT-PCR was performed following the method described previously [35]. After treatment with morphine, the lumbar enlargement of the spinal dorsal horn from rats and mice were harvested for quantitative real-time RTPCR. Total RNA was extracted via the Trizol method (Invitrogen/ThermoFisher Scientific). Reverse transcription was performed using oligo-dT primers and PrimeScriptIIRTase (TAKARA) according to the manufacturer's protocol. Each sample was run in triplicate in a $20 \mu \mathrm{L}$ reaction with $10 \mu \mathrm{M}$ each of forward and reverse primers, $10 \mu \mathrm{l}$ of SYBR Green qPCR Super Mix (Invitrogen) and 25 ng of cDNA. Reactions were performed in an Applied Biosystems 7500 Fast Real-Time PCR System. GAPDH was used as an internal control for normalization. The relative expression ratio of HMGB1 mRNA to GAPDH mRNA in spinal tissues was quantified by the $2-\Delta \Delta^{\mathrm{CT}}$ method. The mouse and rat-specific primer sequences used to detect HMGB1 mRNA and GAPDH mRNA in the qPCR analysis were as follows:

(1) Mice HMGB1 forward, 5'-CGAGCATCCTGGCT TATC-3'; reverse, 5'-ATCCTCCTCATCATCTTCCT-3'. (2) mice GAPDH forward, 5'-AACTTTGGCATTGTGGAAGG -3'; reverse, 5'-GGATGCAGGGATGATGTTCT -3'. (3) rat HMGB1 forward, 5'-TCCTTCGGCCTTCTTCTTGT-3'; reverse, 5'-CGGCCTTCTTTTCATAGGGC-3'. (4) rat TLR4 forward, 5'- GGGGGGTATTTGACACACTCTA-3'; reverse, 5'- TCCTTTGGATGTCTCTATGCGA-3'. (5) rat RAGE forward, 5'-GACCATATGCACAGAAACG-3'; reverse, 5'TAGGGCTGCTTAATAGAC-3'. (6) rat GAPDH forward, 5'TTCACCACCATGGAGAAGGC-3'; reverse, 5'-TGCA GGGATGATGTTCTGGG-3'

\section{Primary Spinal Cord Neuronal Culture}

Primary spinal cord neuronal cultures were established as described previously [26]. Briefly, the spinal cords were separated from rat embryos at gestational day 12-14 under a surgical microscope. Approximately 8 to 10 spinal cords were combined in a $15-\mathrm{ml}$ centrifuge tube and digested in $37^{\circ} \mathrm{C}$ Hanks solution containing $0.2 \%$ trypsin and $0.06 \%$ Dnase for $10 \mathrm{~min}$. The reaction was then terminated with $0.1 \%$ trypsin inhibitor. After filtration through a cell- filter, the cells were suspended in neurobasal medium (Gibco) containing 20\% B27 (Gibco), $2 \mathrm{mM}$ glutamine, and $100 \mathrm{U} / \mathrm{ml}$ penicillin/streptomycin, and seeded in poly-D-lysine coated 6-well plates at a density of $1.5 \times 10^{6}$ cells $/ \mathrm{ml}$. Then, the cells were cultured at $37^{\circ} \mathrm{C}$ in an incubator containing $5 \% \mathrm{CO}_{2}$ and $95 \% \mathrm{O}_{2}$. The cultures were treated with $10 \mu \mathrm{g} / \mathrm{ml}$ cytosine- $\beta$-arabinofuranoside (SigmaAldrich) on days 4 and 5 to prevent the proliferation of nonneuronal cells. The neuron purity was examined by double immunofluorescence staining in which the cells were labeled 
with the neuronal marker MAP2, the astrocytic marker GFAP, and the microglial marker OX42. The results showed that the purity of the neurons reached to $88.7 \%$ on day 11 . All experiments were performed after plating on day 11 . For the dosedependent experiment, different doses of morphine were added to media to reach desired concentration $(0,10,20,40$, 80 , and $160 \mu \mathrm{M})$. After the cells were cultured for 12 hours, the media and the cultured neurons were harvested. For the time-dependent experiment, the $20 \mu \mathrm{M}$ of morphine concentration was used. The cells were cultured for 3, 6, 12, 24, or 48 hours. Then, the media and the cultured neurons were collected. The levels of HMGB1 in media were measured by ELISA. The expression of HMGB1 in cultured neurons was detected by Western blot assay. The concentration of morphine in media was determined by the dose of i.t. injection $(10 \mu \mathrm{g}$ morphine in $10 \mu \mathrm{l}$ saline is approximately $9.8 \mu \mathrm{M}$ morphine) and our previous published paper (26). To assess the quantity of HMGB1 passively released from dead cells, the neuronal death assay was performed one day before morphine exposure and immediately after the end of morphine treatment by Hoechst/propidium iodide costaining of the cultures to distinguish the live and /dead cells. Neuronal cultures were treated with $5 \mu \mathrm{M}$ Hoechst 33342 and $2 \mu \mathrm{M}$ propidium iodide to stain the total and dead cells, respectively. Pictures were taken, and the cells were counted using ImageJ software (NIH).

\section{Fluorescence in Situ Hybridization (FISH)}

FISH was carried out following the method described previously $[26,36]$ by using the rat TLR4 FISH Assay Kit (Boster Bio-Tech, Wuhan, China). The probe used for TLR4 mRNA was labeled with digoxigenin as follows: 5'(DIG)-TTgTgCCCTgTgAggTTgAggTTAg-3'. Briefly, the spinal neurons isolated from rat embryos were seeded onto coverslips in a 6-well plate and cultured at $37^{\circ} \mathrm{C}$ in an incubator containing $5 \% \mathrm{CO}_{2}$ and $95 \% \mathrm{O}_{2}$ for 11 days. After fixation with $4 \%$ paraformaldehyde for $15 \mathrm{~min}$, the coverslips were washed twice with DEPC-PBS and then treated with $10 \mu \mathrm{g} / \mathrm{mL}$ proteinase $\mathrm{K}$ at $37^{\circ} \mathrm{C}$ for $10 \mathrm{~min}$. Then, the coverslips were washed in DEPC-PBS, rinsed with DEPC- $\mathrm{H}_{2} \mathrm{O}$, and prehybridized with ready-to-use prehybridization solution for 3 hours at $50^{\circ} \mathrm{C}$. The DIGlabeled probes were diluted in hybridization buffer and applied at a concentration of $4 \mathrm{ng} / \mu \mathrm{L}$. The coverslips were incubated at $45^{\circ} \mathrm{C}$ for $16 \mathrm{~h}$. Posthybridization washing and immunological detection using anti-DIG-HRP and Tyramide Signal Amplification with cyanine 3 (TSA ${ }^{\mathrm{TM}_{-}}$ Cy3; Perkin Elmer, Waltham, MA, USA), were performed as recommended by the manufacturer. Finally, the coverslips were rinsed with distilled $\mathrm{H}_{2} \mathrm{O}$ and then immunolabeled for NeuN using a FITC-conjugated secondary antibody as described above. Red fluorescence indicates TLR4 mRNA; green fluorescence indicates immunohistochemical staining for neurons.

\section{siRNA Transfection}

siRNA transfection was carried out following our previously described method [26]. The HMGB1 small interfering RNA (siRNA) target rat hmgb1 was purchased from OriGene (OriGene Technologies, Rockville, SR506455). A universal scramble negative control RNA duplex was also provided by OriGene. The TurboFect in vivo transfection reagent (Thermo Scientific Inc.) was used as a delivery vehicle for the siRNA to prevent degradation and enhance the cell membrane penetration of the siRNA. Rats were injected intrathecally with siRNA (at a dose of $0.1 \mathrm{nmol} / 10 \mu \mathrm{l}$ ) or vehicle (transfection regent) one hour before morphine administration once daily for 5 days. The sequences of rat HMGB1 siRNA u s e d we re a s follow s : 5'-C A G G AGGAAUACUGAACAUdTdT-3' (sense) and 3'-dTdT GUCCUCCUUAUGACUUGUA-5' (antisense).

The rat TLR4 siRNA was purchased from Santa Cruz (Santa Cruz Biotechnology, Inc. SC-1560001). Once the spinal cord neurons from rat embryos had been cultured for 11 days, the cells were transfected with TLR4 siRNA or scramble RNA (scRNA) (Santa Cruz Biotechnology) at a final concentration of $50 \mathrm{nM}$ using Lipofectamine 2000 (Invitrogen) according to the manufacturer's instructions. Twelve hours after transfection, the cells and media were harvested, and the effect of siRNA on protein expression and HMGB1 release was determined by Western blot and ELISA. TLR4 siRNA delivery to the spinal cord was performed following the same method as that used for the HMGB1 siRNA i.t. injections.

\section{Enzyme-linked immunosorbent assay (ELISA)}

ELISA was performed following the method described previously [37]. After the spinal neurons were cultured for the designated amount of time, the media were collected in Eppendorf tubes and stored at $-80^{\circ} \mathrm{C}$. The HMGB1 level was tested with a rat HMGB1 ELISA kit (CSB-E08224r, CUSABIO, China) according to the manufacturer's instructions. A total of $100 \mu \mathrm{l}$ of each diluted standard substance was added to a 96-well plate to prepare a standard curve, and $100 \mu \mathrm{l}$ of each sample was added to each well, and the samples were tested in triplicate. After incubation with a biotinantibody, HRP-avidin, and TMP substrate, $50 \mu \mathrm{l}$ of stop buffer was added sequentially, and the absorbance was measured at a wavelength of $450 \mathrm{~nm}$ with a microplate reader (ELx808, Bio Tec, USA). The linear regression equation of the standard curve was calculated according to the concentrations of the standards and the OD value. The sample concentrations were calculated according to the standard curve. 


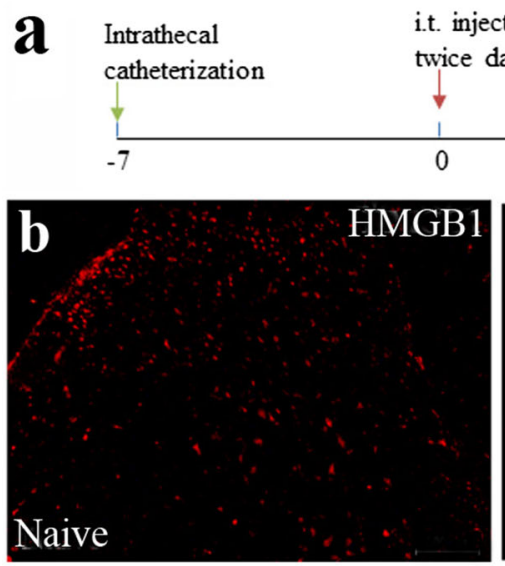

i.t. injection of morphine

Harvest spinal cord for

$\mathrm{IHC}, \mathrm{WB}$, and $\mathrm{qPCR}$

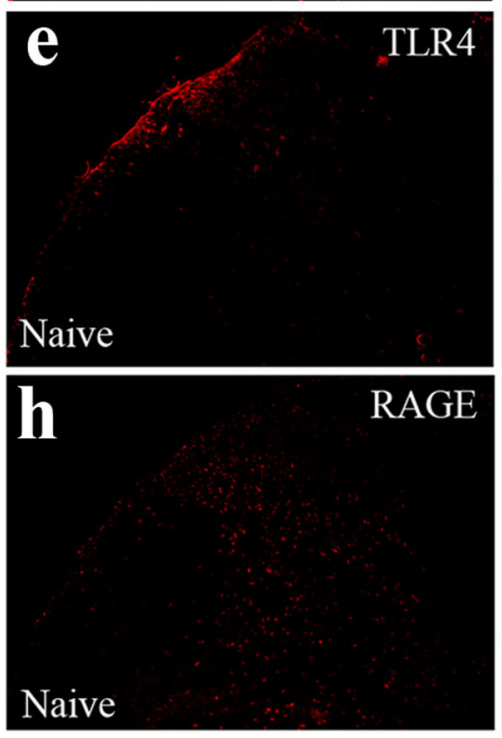

Sa i.t.

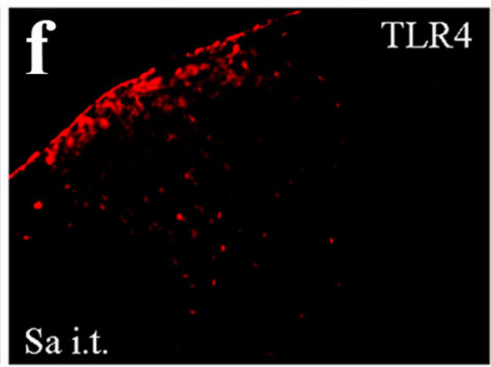

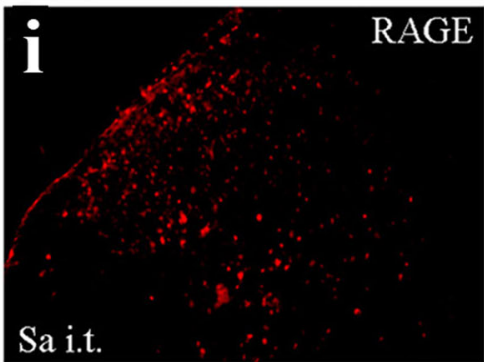

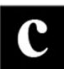

Mor 1.t.

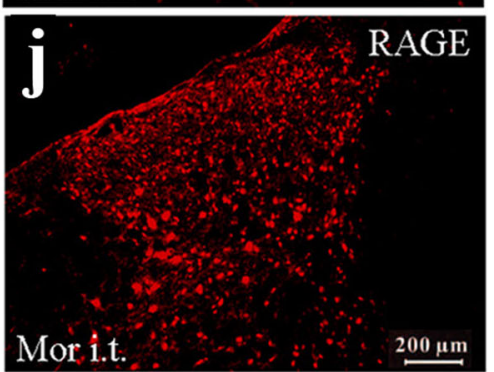

k

Control $1 \quad 3 \quad 5 \quad 7 \quad 10 \mathrm{~d}$
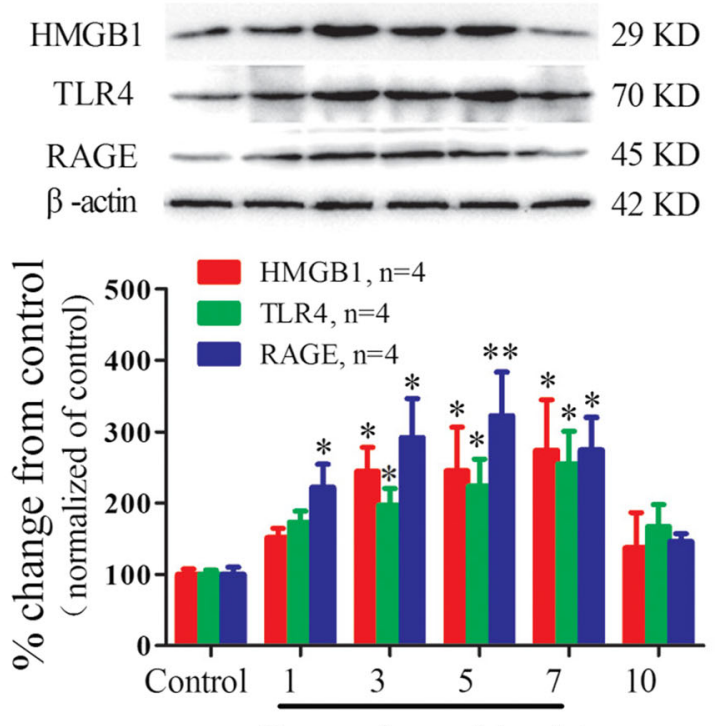

Days of morphine i.t.
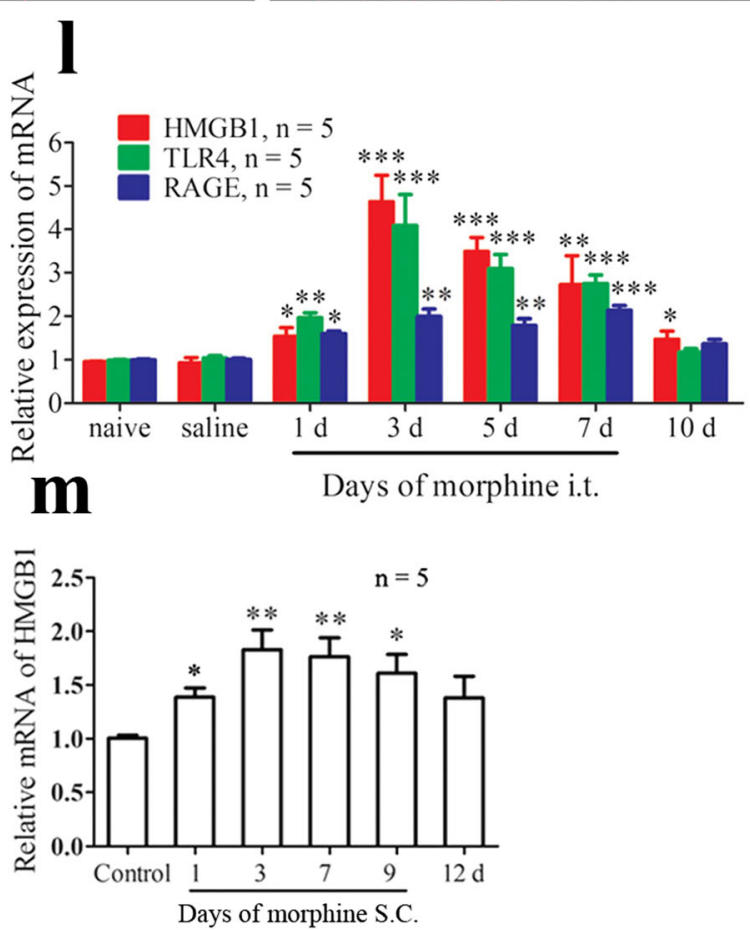
Fig. 1 The expression of HMGB1, TLR4, and RAGE proteins and mRNA in the spinal dorsal horn. (a) Diagram of the timeline of this experiment. (b-d) Images showing HMGB1 expression in the spinal dorsal horn of naïve rats (b) and rats receiving intrathecal (i.t.) injection of saline (c) and i.t. injection of morphine (d). (e-g) Images showing TLR4 expression in the spinal dorsal horn of naïve rats (e) and rats receiving i.t. injection of saline (f) and i.t. injection of morphine (g). (gh-j) The images showing RAGE expressed in spinal dorsal horn in rats of naïve rats (h) and rats receiving i.t. injection of saline (i) and i.t. injection of morphine (j). (k) Repeated intrathecal (i.t.) injections of morphine led to a significant increase in the expression of HMGB1, TLR4, and RAGE proteins in the spinal dorsal horn. $* P<0.05$, $* * P<$ 0.01 vs. control group (i.t. injection of saline daily for 6 days). (l) Repeated i.t. injections of morphine led to a significant increase in the expression of HMGB1, TLR4, and RAGE mRNA in the spinal dorsal horn. $* P<0.05$, ** $P<0.01$, *** $P<0.001$ vs. saline group (i.t. injection of saline daily for 6 days). (m) Repeated subcutaneous (s.c.) injections of morphine caused increased expression of HMGB1 mRNA in the spinal dorsal horn of mice. * $P<0.05$; ** $P<0.01$ vs. control (s.c. saline daily for 9 days). Data are presented as the mean \pm SEM and were analyzed with one-way ANOVA. Images in $\mathbf{b}-\mathbf{j}$, scale bar $=200 \mu \mathrm{m}$. Sa: saline

\section{Statistical analysis}

Statistical analyses were performed with Prism 4.02 (GraphPad Software, USA) and SigmaStat (Systat, San Jose, CA). The sample sizes were determined based on the power analysis of a preliminary experiment and the experience of our previous similar studies. All data are presented as the mean \pm SEM. For behavioral data, differences over time were tested using two-way ANOVA with repeated measures followed by Tukey's post hoc test, and one-way ANOVA followed by individual Tukey's post hoc comparisons was carried out for the data between groups at the same time point. The differences in Western blot and qPCR data were evaluated by oneway ANOVA followed by Tukey's post hoc tests or Student's $t$-test if only two groups were applied. The dose- and timedependent morphine-stimulated expression and release of morphine in cultured spinal neurons were analyzed by twoway ANOVA followed by Tukey's post hoc test. $P<0.05$ was considered significant in all tests.

\section{Results}

\section{Chronic intrathecal injections of morphine increase the expression of HMGB1, TLR4, and RAGE in the rat spinal dorsal horn}

A previous study revealed that TLR4 and RAGE function as receptors for HMGB1 in the nervous system [9]. Therefore, the expression levels of HMGB1, TLR4, and RAGE in the spinal dorsal horn were examined by immunofluorescence staining, Western blot, and real-time quantitative PCR (qPCR) following repeated i.t. administrations of morphine. Compared to the saline group and naïve rats, rats receiving repeated i.t. injections of morphine exhibited markedly increased immunoreactivity (IR) for HMGB1 (Fig. 1b-d), TLR4 (Fig. 1e-g), and RAGE (Fig. 1h-j) in the spinal dorsal horn. Western blot results showed a significant increase in the expression of HMGB1, TLR4, and RAGE in the dorsal horn of the lumbar enlargement segments. A significant difference in HMGB1 expression compared to that in the rats in the control group (which received saline i.t. for 6 days) occurred at day 3 and persisted until day 7 of treatment in the rats receiving morphine injections $(* P<0.05$ vs. control, Fig. $1 \mathrm{k})$. Chronic i.t. morphine exposure also increased the expression of TLR4 and RAGE in the spinal cord. A significant increase in TLR4 and RAGE expression compared to that in the rats in the control group occurred on day 1 , peaked on day 5 , and lasted until day $7(* P<0.05$, ** $P<0.01$, Fig. $1 \mathrm{k})$ of treatment. Moreover, morphine exposure also led to an increase in the expression of HMGB1 in L4 and L5 DRGs (data not shown). To further verify the above protein changes, the mRNA expression levels of HMGB1, TLR4, and RAGE in the dorsal horn of the lumbar enlargement were examined by qPCR. Compared to the rats in the i.t. saline group and the naïve rats, rats receiving chronic i.t. morphine exposure exhibited a significant increase in the expression of HMGB1, TLR4, and RAGE mRNA, which occurred on day 1, peaked on day 3 , and persisted until day 7 of the morphine injections (* $P<0.05$, ** $P<0.01$, *** $P<0.001$ vs. saline group. Fig. 11). To exclude the results caused by possible inflammatory reactions following repeated i.t. injections, repeated subcutaneous (s.c.) injections of morphine in mice $(15 \mathrm{mg} / \mathrm{kg}$, once daily for 9 days) were performed to observe morphineinduced HMGB1 mRNA expression in the spinal cord. The results of the qPCR assay showed that, s.c. morphine led to significantly increased expression of HMGB1 mRNA in the dorsal horn compared to that induced by the control treatment (saline s.c.), which started at day $1(P<0.05)$, peaked at day 3 $(P<0.01)$, and persisted until day $9(P<0.05$, Fig. $1 \mathrm{~m})$ of morphine injections. Taken together, these results indicate that chronic i.t. morphine exposure stimulates the expression of HMGB1, TLR4, and RAGE in the spinal dorsal horn.

Based on the above findings, double immunofluorescence staining was performed to examine the cell types that express HMGB1, TLR4, and RAGE in the spinal cord in rats that receive i.t. administration of morphine and vehicle (saline) alone. In vehicle-treated group, the results showed that there was somewhat expression of HMGB1 in spinal neurons, astrocytes, and microglia (S1a-i), and a little TLR4 expressed in astrocytes and microglia (S2a-i), and RAGE slightly colocalized with NeuN and GFAP (S3a-i). However, in morphine-treated group, the results revealed that HMGB1 colocalized with the neuronal marker NeuN (Fig. 2a), the astrocytic marker GFAP (Fig. 2b), and the microglial marker OX42 (Fig. 2c). TLR4 also colocalized with NeuN, GFAP, and OX42 (Fig. 2d-f). However, RAGE colocalized with 

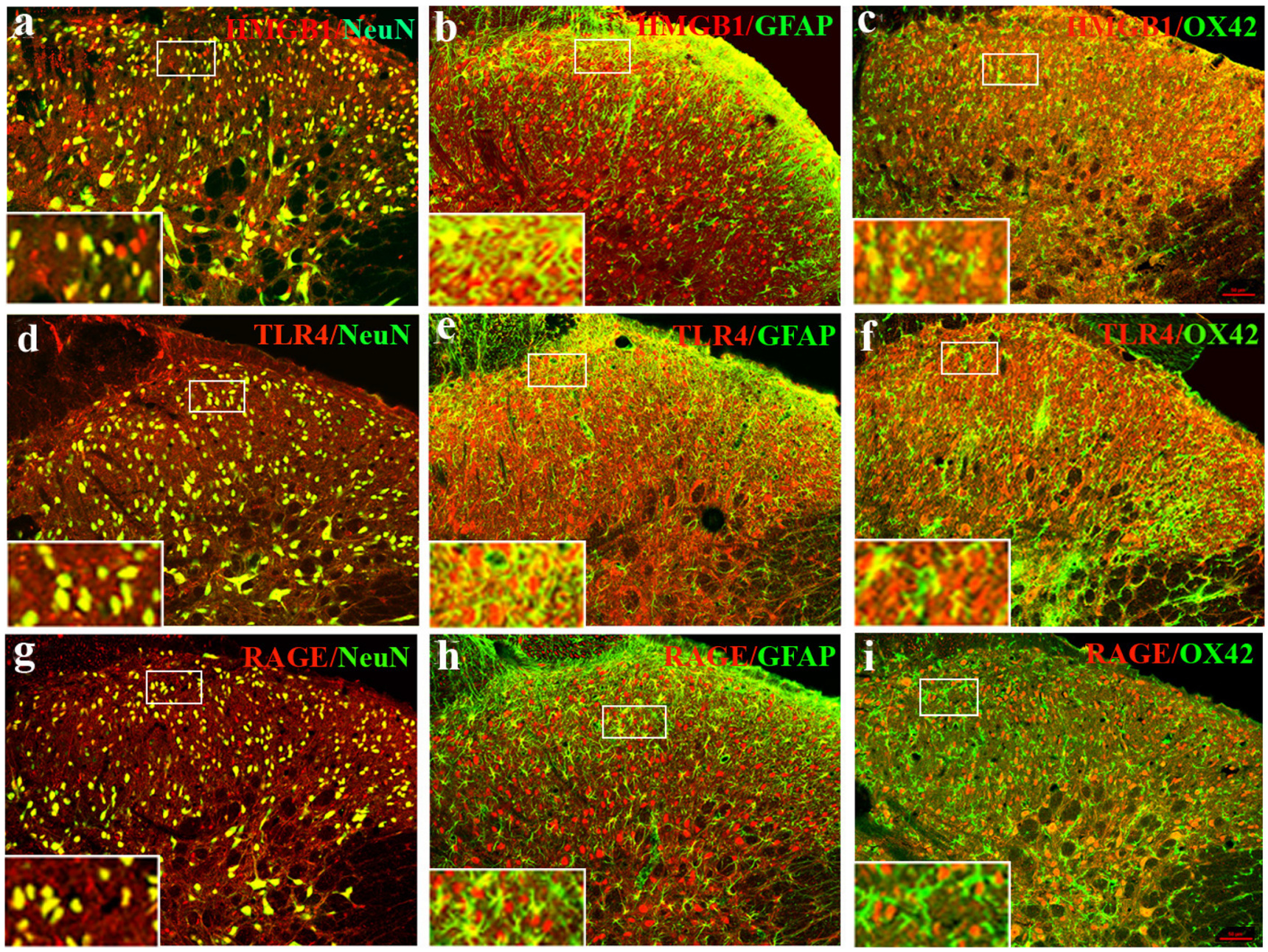

Fig. 2 The cell types that express HMGB1, TLR4, and RAGE in the rats spinal dorsal horn following repeated intrathecal (i.t.) injections of morphine. (a-c) Representative images showing that HMGB1 colocalized with the spinal neuronal marker NeuN (a), the astrocytic marker GFAP (b), and the microglial marker OX42 (c). (d-f)

Representative images showing TLR4 colocalization with NeuN (d), GFAP (e), and OX42 (f). (g-i) Representative images showing RAGE colocalization with NeuN (g) and GFAP (h) but not with OX42 (i). Images in $\mathbf{a}-\mathbf{i}$, scale bar $=50 \mu \mathrm{m}$.

NeuN extensively (Fig. 2g) and slightly with GFAP (Fig. 2h), but not with OX42 (Fig. 2i). The separated images for merged HMGB1, TLR4, and RAGE were provided in supplemental data S4, S5, and S6.

\section{Morphine exposure increases the expression and release of HMGB1 in primary cultured spinal neurons}

Given that chronic i.t. morphine exposure significantly increased the expression of HMGB1 and TLR4 in spinal neurons and glial cells, spinal neurons from fetal rats were cultured to observe the direct effect of morphine exposure on the expression and release of HMGB1. First, the purity of the cultured spinal neurons was examined by double immunofluorescence staining. The results showed that the percentage of neurons in the culture reached $88.7 \%$ (Fig. 3b-g). Second, the expression of TLR4 protein and mRNA in the cultured

neurons was examined because TLR4 plays an important role in the active release of HMGB1. The results showed that both the TLR4 protein (Fig. 3h-j) and mRNA (Fig. 3k-m) colocalized with the neuronal marker NeuN. Finally, the morphine-stimulated expression and release of HMGB1 were examined. The Western blot results showed that morphine challenge resulted in an increase in the expression of HMGB1 in the cultured spinal neurons. The significant differences in HMGB1 expression in the treatment groups in the dose-dependent experiment compared to that in the control treatment (in which saline was added to the medium) occurred at a concentration of $10 \mu \mathrm{M}$ of morphine $(P<0.05)$, peaked at $20 \mu \mathrm{M}(P<0.01)$, and persisted to $160 \mu \mathrm{M}(P<0.05)$ (twoway ANOVA, Fig. 3n). In another experiment, the cultured neurons were challenged with $20 \mu \mathrm{M}$ morphine for different amounts of time. The results showed an increase in HMGB1 expression compared to that in the control cells (which received saline added to the medium) started at 3 hours $(P<$ 

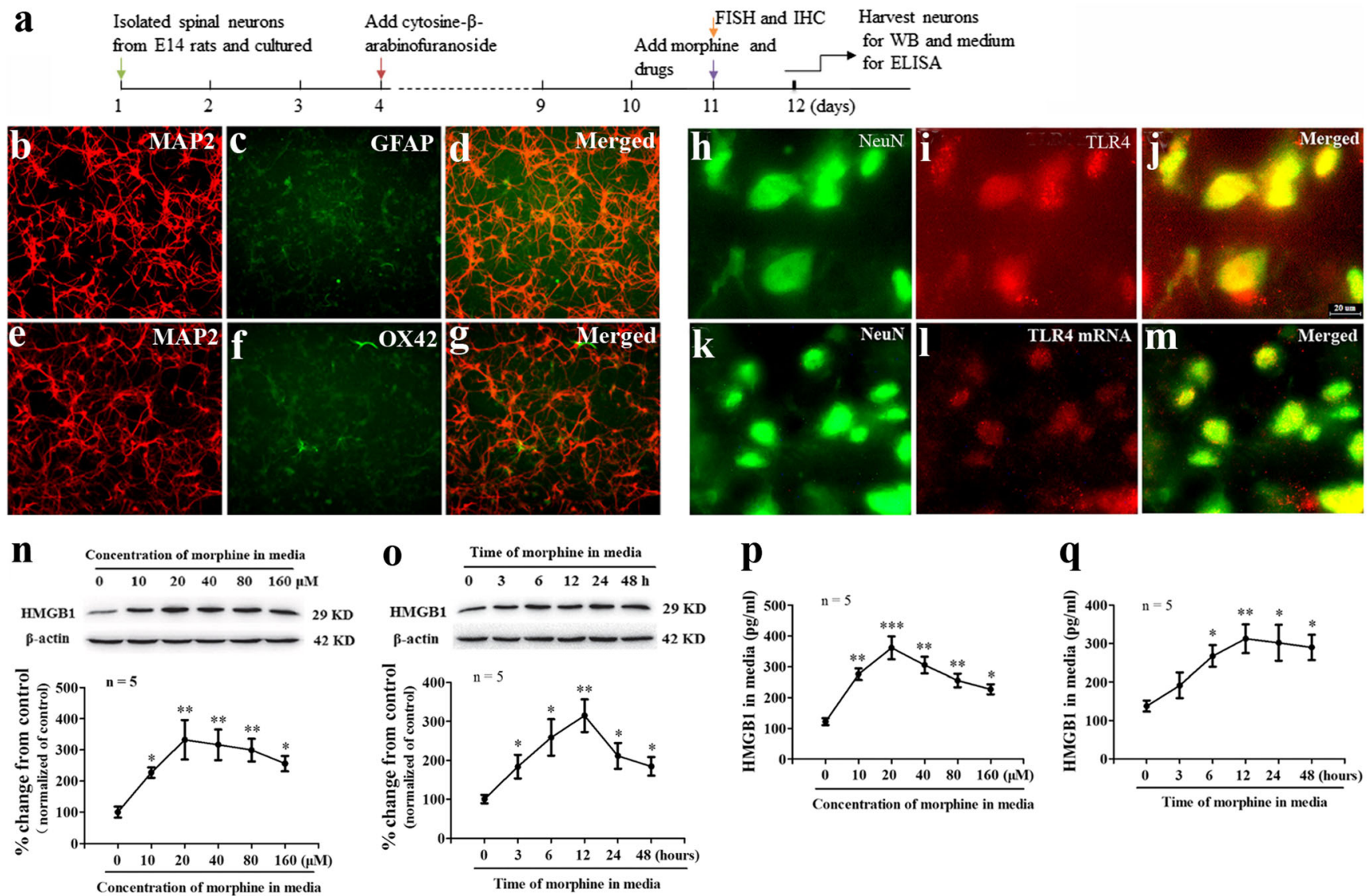

Fig. 3 Effects of morphine exposure on the expression and release of HMGB1 in primary cultured spinal neurons. (A) Diagram of the timeline of this experiment. (b-g) The cultured neuron purity was calculated by labeling neurons with the neuronal marker MAP2 and glial cells with GFAP (an astrocytic marker) and OX42 (a microglial marker). The images were merged in (d) and (g). Scale bar $=50 \mu \mathrm{m}$. (h-j) Representative images showing the colocalization of the neuronal marker NeuN (h) with the TLR4 protein (i) in cultured cells (j). (k-m) Images showing the neuronal marker NeuN (k) colocalized with TLR4 mRNA (i) in cultured cells (m). Images in $\mathbf{h}-\mathbf{m}$, scale bar $=50 \mu \mathrm{m}$. $(\mathbf{n}, \mathbf{o})$ Morphine exposure

$0.05)$, peaked at 12 hours $(P<0.01)$, and lasted until 48 hours $(P<0.05)$ of morphine stimulation (two-way ANOVA, Fig. 3o). Because HMGB1 act as proinflammatory mediator only when present extracellularly, the concentration of HMGB1 in the media was examined by ELISA. The results showed that morphine challenge resulted in an increase in the release of HMGB1 in the media in a dose- and time-dependent manner (Fig. 3p and q). The significant increase in HMGB1 levels in the media compared to those in the media of the control cells (which received saline added to the media) occurred at a concentration of $10 \mu \mathrm{M}$ morphine $(P<0.01)$, peaked at $20 \mu \mathrm{M}(P$ $<0.001)$, and persisted to $160 \mu \mathrm{M}(P<0.05)$ (two-way ANOVA, Fig. 3p). In a time-dependent experiment, the significant increase in HMGB1 levels in cells treated with $20 \mu \mathrm{M}$ morphine compared to those in media of the control cells occurred at 6 hours $(P<0.05)$, peaked at 12 hours $(P<$ $0.01)$, and persisted until 48 hours $(P<0.05)$ of treatment promoted the expression of HMGB1 in primary cultured spinal neurons in a dose- (neurons were cultured with different concentrations of morphine for $12 \mathrm{~h}$ ) (n) and time- (neurons were cultured in medium containing $20 \mu \mathrm{M}$ morphine for different amounts of time) (o) dependent manner. $* P<0.05$; $* * P<0.0$; *** $P<0.001$ vs. control group. $(\mathbf{p}, \mathbf{q})$ Morphine challenge resulted in the increased release of HMGB1 from cultured neurons in a dose- $(\mathbf{p})$ and time- $(\mathbf{q})$ dependent manner. $* P<$ $0.05 ; * * P<0.01$; *** $P<0.001$ vs. control group. Data are presented as the mean \pm SEM and were analyzed with two-way ANOVA.

(two-way ANOVA, Fig. 3q). Although the main source of HMGB1 may be the expression and release of the protein from spinal glial cells in vivo, the above results provide direct evidence that morphine stimulation leads to the increased expression and release of HMGB1.

\section{Intrathecal glycyrrhizin, an inhibitor of HMGB1, and HMGB1 siRNA impair the development of morphine-induced analgesic tolerance and hyperalgesia}

We further evaluated the role of spinal HMGB1 in the development of morphine tolerance and morphine withdrawalinduced hyperalgesia. As previously reported [26], repeated twice-daily intrathecal injections of $10 \mu \mathrm{g}$ morphine plus vehicle (saline) for 6 days led to a time-dependent decrease in morphine's maximal potential analgesic effect (MPAE). The 
MPAEs on days 3, 5, and 7 postinjection compared with those on day one were reduced by $2.0 \%, 73.2 \%$, and $96.31 \%$, respectively (Fig. 4b). Because recent studies have shown that glycyrrhizin (GL) works as an HMGB1 inhibitor and can bind directly to HMGB1 and inhibit its extracellular cytokine activities [20, 21, 38], i.t. coinjections of morphine with GL were performed. The results showed that the reduction of morphine's MPAE was prevented by the once-daily coadministration of GL with morphine. Compared with that in the morphine plus vehicle group, the MPAE in the morphine plus GL groups decreased $69.2 \%$ at a GL concentration of $10 \mu \mathrm{g}$, $48.4 \%$ at $20 \mu \mathrm{g}$, and $23.42 \%$ at $40 \mu \mathrm{g}$ of GL on day 7 (Fig. $4 \mathrm{~b})$. The basal MPAE value was not changed when rats received GL (40 $\mu \mathrm{g} / \mathrm{d}$, i.t.) alone. Consistent with previous reports, 6 days of repeated intrathecal morphine injections induced mechanical and thermal pain hypersensitivities on day 8 , one day after morphine withdrawal. The paw withdrawal thresholds (PWTs) in response to mechanical stimuli were reduced by $63.1 \%$ and $64.7 \%$ in the left and right hand paws, respectively, compared with those at baseline, and paw withdrawal latencies (PWLs) in response to thermal stimuli were reduced by $49.72 \%$ and $47.28 \%$ in left and right hind paws, respectively (data for the right paw are not shown, Fig. $4 \mathrm{c}$ and d). These reductions were partially prevented by the coadministration of GL dose-dependently. The coinjection of GL clearly reduced the decrease in the PWTs (Fig. 4c) and PWLs compared to that observed upon control treatment (morphine plus vehicle) (Fig. 4d). GL alone did not alter basal paw withdrawal responses to mechanical and thermal stimuli (Fig. 4c and $\mathrm{d}$ ).

To further confirm our pharmacological results, repeated i.t. injections of HMGB1 siRNA were performed to inhibit HMGB1 expression in the spinal cord. Behavioral data showed that the reduction of the MPAE following 6 days of i.t. morphine administration was dramatically prevented by coinjections of HMGB1 siRNA once daily for 5 days. Compared with that in the morphine plus vehicle and morphine plus scramble RNA groups, the MPAE in the morphine plus HMGB1 siRNA group decreased $33.5 \%$ on day $7(P<$ 0.01 , Fig. $4 \mathrm{e}$ ). The morphine withdrawal-induced mechanical allodynia and thermal hyperalgesia were also alleviated in rats that received HMGB1 siRNA treatment (left PWT: $P<0.001$ vs. morphine plus vehicle or morphine plus scRNA, Fig. 4f; left PWL: $P<0.05$ vs. morphine plus vehicle or morphine plus scRNA, Fig. 4g). Repeated i.t. injections of HMGB1 siRNA or vehicle (transfection reagent) alone did not change the basal tail-flick response to thermal stimulation and morphine-induced hyperalgesia (Fig. 4e-g). Western blot data showed that the level of HMGB1 protein in the L4-6 spinal cord in the morphine plus vehicle group increased 2.12-fold compared to that in the vehicle alone group. This increase was clearly inhibited by the coadministration of HMGB1 siRNA (morphine plus vehicle vs. morphine plus HMGB1 siRNA: $P$
$<0.001)$ but not by HMGB1 scramble RNA $(P>0.05$, Fig. $4 \mathrm{~h})$. The results of qPCR showed that the i.t. administration of HMGB1 siRNA significantly inhibited the morphine-induced expression of HMGB1 mRNA in the spinal cord (morphine plus vehicle vs. morphine plus HMGB1 siRNA: $P<0.001$, Fig. 4i).

\section{Intrathecal GL and an HMGB1 neutralizing antibody partially reverse established morphine tolerance and hyperalgesia}

We also investigated the role of spinal HMGB1 in the maintenance of morphine tolerance and hyperalgesia. GL or an HMGB1 neutralizing antibody was administered with morphine intrathecally beginning on day 7 (a time point at which morphine tolerance and hyperalgesia have been fully established) of an 11-day course of morphine injections. The results showed that both the GL and HMGB1 neutralizing antibody treatments clearly reversed the morphine-induced reduction in MPAE and the morphine withdrawal-induced decreases in PWTs and PWLs (Fig. 5). Compared with that of the control (morphine plus vehicle) group, the MPAE increased $68.28 \%$ (Fig. 5b), the PWT increased 3.7-fold (Fig. 5c), and the PWL (Fig. 5d) increased by $41.1 \%$ in the morphine plus GL group. Repeated i.t. injections of GL (40 $\mu \mathrm{g}$ ) or saline alone did not change the basal values of the MPAE, PWTs, and PWLs (Fig. 5b-d). In the group of rats treated with the HMGB1 neutralizing antibody $(2 \mu \mathrm{g}$, once daily for 3 days), the MPAE increased by $43.74 \%$ (Fig. 5e), the PWT increased 2.3-fold (Fig. 5f), and the PWL (Fig. 5g) increased $42.8 \%$, compared to the values in the rats treated with morphine plus vehicle or with morphine plus control IgG. The basal values of the MPAE, PWTs, and PWLs were not changed in rats that received the HMGB1 neutralizing antibody or ACSF i.t. injection alone (Fig. 5e-g).

\section{The role of spinal HMGB1 in morphine tolerance and morphine withdrawal-induced hyperalgesia in female rats}

To compare the effect of spinal HMGB1 in morphine tolerance between male and female rats, the expression of HMGB1, TKLR4, and RAGE in the spinal dorsal horn in female rats were also examined following 6 days of repeated i.t. injections of morphine. As observed in male rats, the morphine treatment also elicited significant increase in the expression of HMGB1, TKLR4, and RAGE in the spinal dorsal horn (Fig. 6a). Compared with control group, the statistical difference occurred on day 1 , peaked on day 5 , and persisted until stopping morphine treatment $(* P<0.05$, ** $P<0.01$, Fig. $6 a)$. Behavioral data assay showed that the repeated injections of morphine resulted in significant reduction of morphine's MPAE at day 7 . But this decrease was partially prevented by 


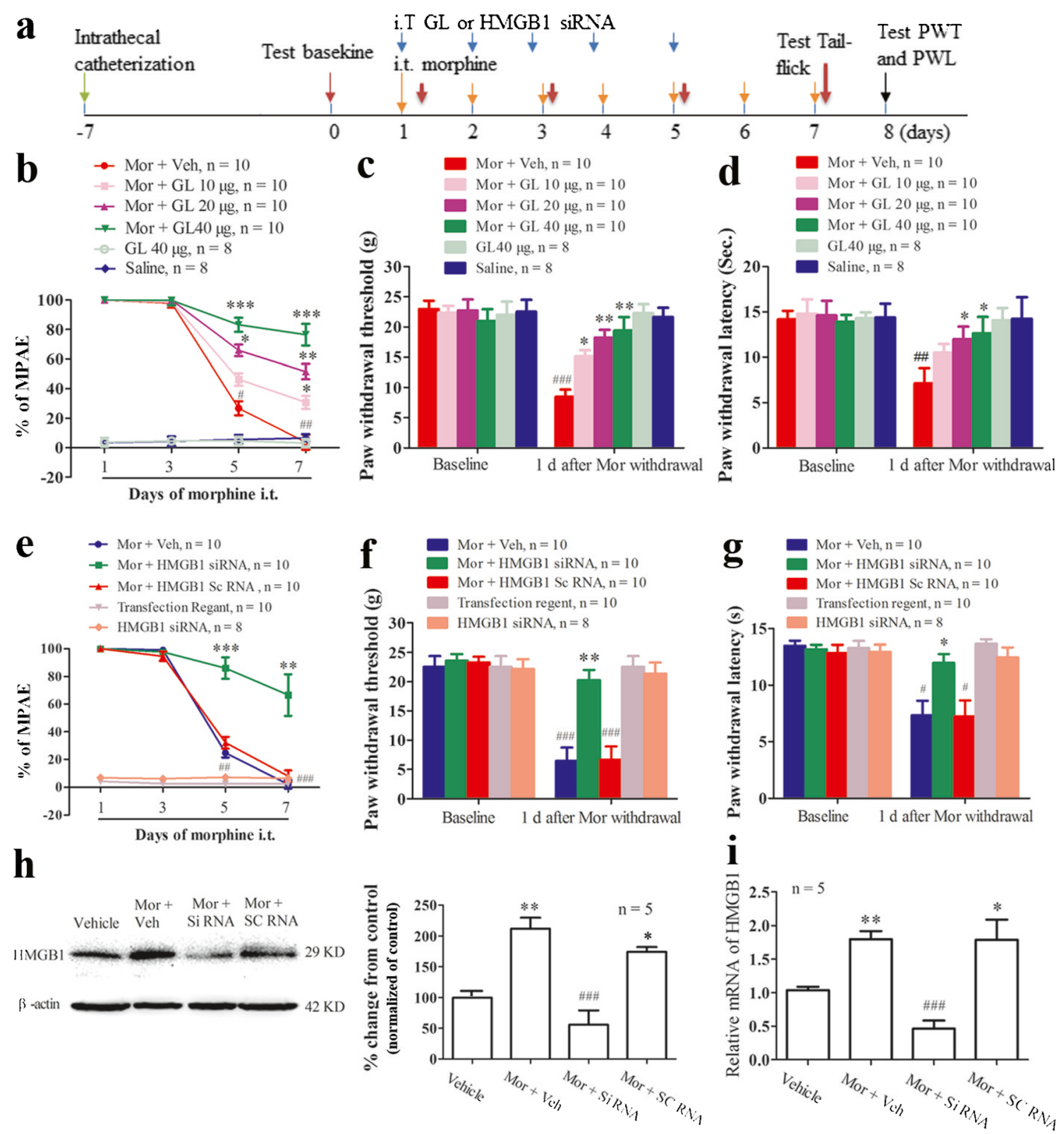

Fig. 4 The role of the chronic i.t. morphine exposure-induced upregulation of HMGB1 in the spinal dorsal horn in the development of analgesic tolerance and hyperalgesia. (a) Diagram of the timeline of this experiment. (b) Repeated i.t. injections of morphine led to a significant reduction in morphine's maximal possible analgesic effect (\% MPAE). \# $P<$ 0.05 ; \#\# $P<0.01$ vs. day one. Data are presented as the mean $\pm \mathrm{SEM}$ and were analyzed by two-way ANOVA. Intrathecal coadministration of morphine (Mor) plus glycyrrhizin (GL), an inhibitor of HMGB1, dosedependently prevented the decrease in the MPAE. * $P<0.05$; ** $P<$ 0.01 ;*** $P<0.001$ vs. morphine plus vehicle (Veh) group (one-way ANOVA). (c, d) Chronic i.t. morphine exposure resulted in decreased paw withdrawal threshold (PWT) (c) and paw withdrawal latency (PWL) (d) in the left hind paw. \#\# $P<0.01$ vs. baseline. Repeated intrathecal coinjections of morphine plus GL prevented the reduction in PWT and PWL after morphine withdrawal. $* P<0.05$; $* * P<0.01$ vs. morphine plus vehicle group (Student's $t$-test). The basal tail-flick response, PWT and PWL were not changed by repeated i.t. injections of GL alone. (e) Repeated i.t. injections of morphine plus vehicle (Veh: transfection regent) or morphine plus scramble (sc) RNA resulted in significant reductions in the MPAE at day 5 and day 7 . \#\# $P<0.01$ vs. day one (two-way ANOVA). This effect was clearly prevented by the coadministration of morphine with HMGB1 siRNA intrathecally. $* * P$ $<0.01$; *** $P<0.001$ vs. morphine plus vehicle or morphine plus HMGB1 scRNA (one-way ANOVA). (f, g) Repeated i.t. injections of morphine plus vehicle or morphine plus HMGB1 scRNA led to significant decreases in PWT (f) and PWL (g) in the left hind paw, but these reductions were prevented by i.t. coinjections of morphine plus HMGB1 siRNA. \# $P<0.05$, \#\# $P<0.01$ vs. baseline; $* P<0.05$, ** $P<0.01$ vs. morphine plus vehicle or morphine plus HMGB1 scRNA (Student's $t$ test). The basal tail-flick response, PWT, and PWL were not changed by repeated i.t. injections of HMGB1 siRNA or transfection regent alone. (h, i) Repeated i.t. coinjections of morphine plus vehicle or morphine plus HMGB1 scRNA led to significantly increased expression of the HMGB1 protein (h) and HMGB1 mRNA (i) in the spinal dorsal horn. These effects were inhibited by i.t. coadministration of morphine with HMGB1 siRNA. $* P<0.05$, ** $P<0.01$ vs. vehicle group; \#\#\# $P<0.001$ vs. morphine plus vehicle or morphine plus scramble RNA (one-way ANOVA). 


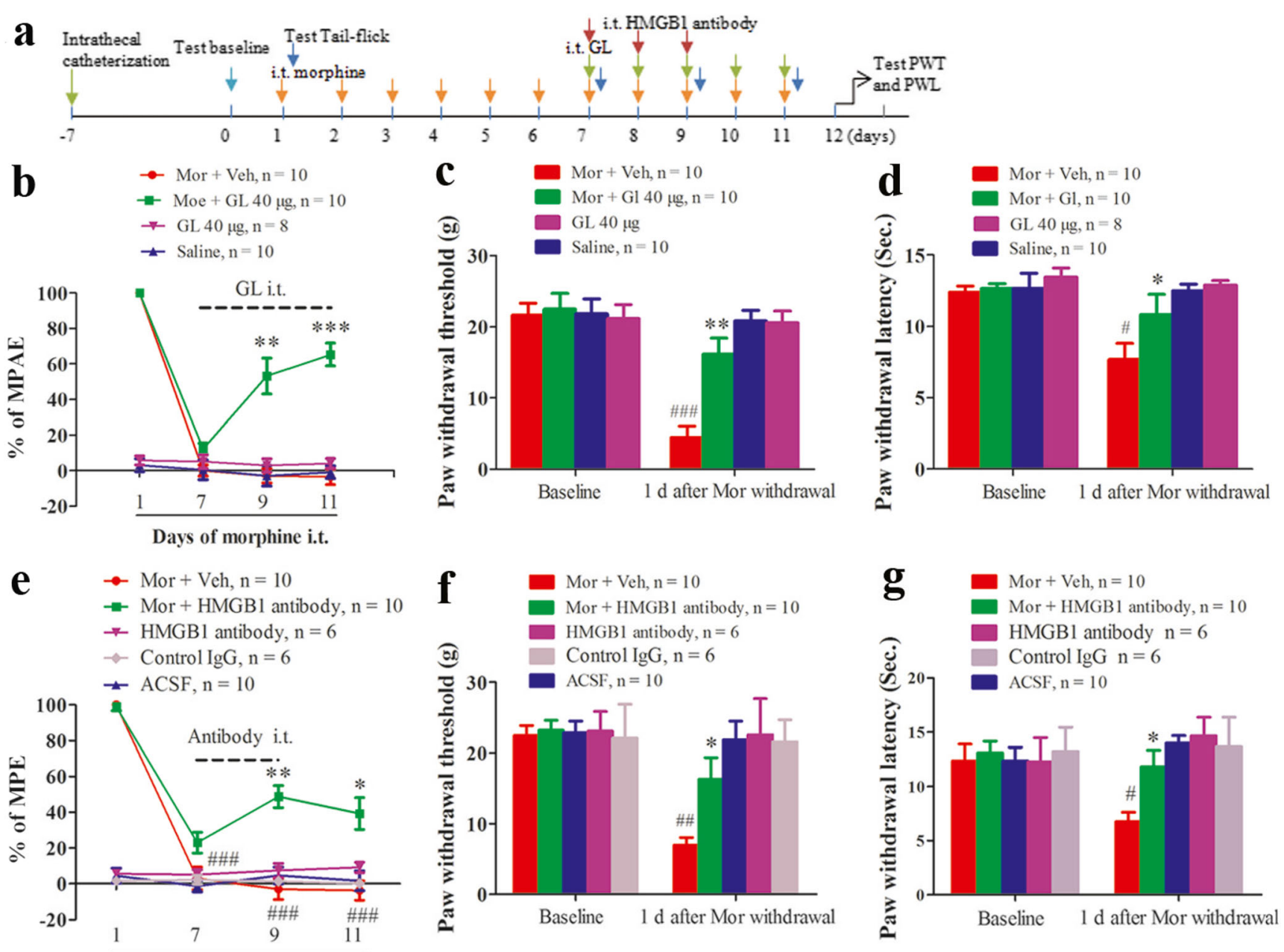

Days of morphine i.t.

Fig. 5 The effects of repeated i.t. administration of glycyrrhizin (GL) and an HMGB1 neutralizing antibody on established morphine tolerance and hyperalgesia. (a) Diagram of the timeline of this experiment. (b-d) Repeated i.t. coinjections of morphine plus vehicle (saline) resulted in a significant decrease in the MPAE on day 7, day 9 and day 11. \#\#\# $P<$ 0.001 vs. day one (two-way ANOVA) (b). This reduction was reversed by the i.t. coadministration of morphine with GL started at day 7. $* * P<$ $0.01, * * * P<0.001$ vs. morphine plus vehicle group (Student's $t$-test). The morphine withdrawal-induced reductions in PWT (c) and PWL (d) in the left hind paw were also partially reversed in the morphine plus GL group. $\# P<0.05$, \#\#\# $P<0.001$ vs. baseline. $* P<0.01$, $* * P<0.01$ vs. morphine plus vehicle group (Student's $t$-test). The basal tail-flick

the pretreatment of i.t. injection of GL (Morphine plus GL vs. Morphine plus Vehicle group, $* * P<0.01$, *** $P<0.001$. \#\# $P<0.01$, \#\#\# $P<0.001$ vs. day 1 . Fig. 6 b). The morphine withdrawal-induced reductions of PWT and PWL were also partially prevented by the treatment $\# P<0.05$, \#\# $P<0.01$ vs. baseline. $* P<0.05$ vs. morphine plus vehicle group. Fig. $6 c, d)$. When i.t. injection of HMGB1 neutralizing antibody started at day 7, a time-point of morphine tolerance has been established fully, the decreased morphine's MPAE and morphine withdrawal-induced reductions of PWT and PWL were also partially reversed $(* P<0.05$, ** $P<0.01$, *** $P<0.001$ response, PWT, and PWL were not changed by repeated i.t. injections of GL or saline alone. (e) The reduction in the MPAE induced by repeated i.t. injections of morphine were also partially reversed by the i.t. coinjection of morphine with an HMGB1 neutralizing antibody starting at day 7. $* P<0.05$, $* * P<0.01$ vs. morphine plus vehicle group (Student's $t$-test). (f, g) Coadministration of morphine with an HMGB1 neutralizing antibody alleviated morphine withdrawal-induced mechanical allodynia (f) and thermal hyperalgesia (g). $* P<0.05$ vs. morphine plus vehicle group (Student's $t$-test). The basal tail-flick response, PWT, and PWL were not changed by repeated i.t. injections of control IgG or ACSF alone. ACSF: artificial cerebrospinal fluid; Mor: morphine; Veh: vehicle.

vs. morphine plus vehicle group. \# $P<0.05$, \#\# $P<0.01$, \#\#\# $P<0.001$ vs. baseline or day 1. Fig. 6e-g).

The morphine withdrawal-induced spontaneous pain was also examined after 7 days repeated i.t. injections of morphine in male (Fig. 6h) and female rats (Fig. 6i), respectively. All rats showed equivalent preconditioning time in the vehicle-, clonidine-, and GL-paired groups, indicating no preconditioning bias for either group (Fig. 6h and i). After rats received vehicle, clonidine, and GL i.t. injection, the times that spent in chambers between vehicle-, clonidine-, and GL-paired group were not reached statistical difference at testing day (Fig. 
$\mathbf{a}$

\section{Control $1 \begin{array}{llllll}1 & 3 & 5 & 7 & 10 & \mathrm{~d}\end{array}$}

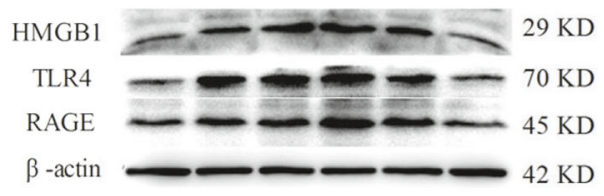

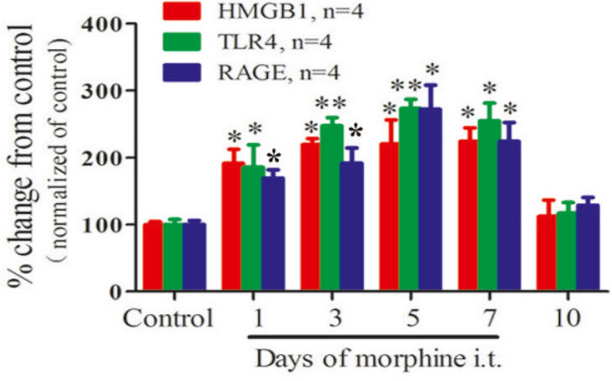

b

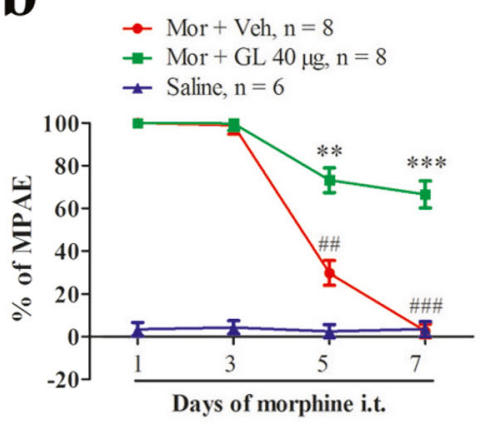

c

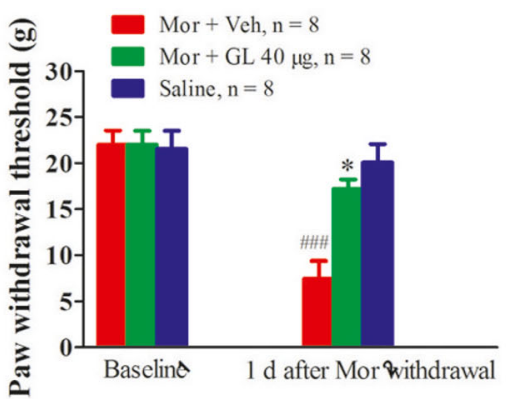

e

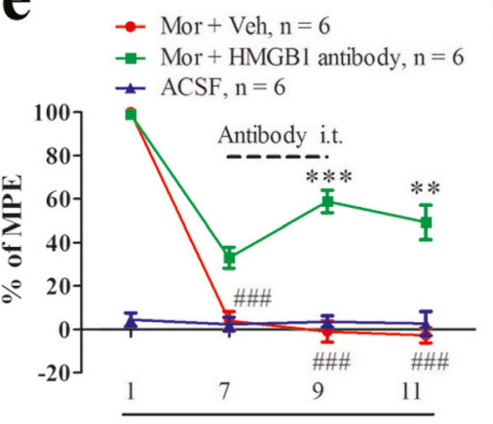

Days of morphine i.t.

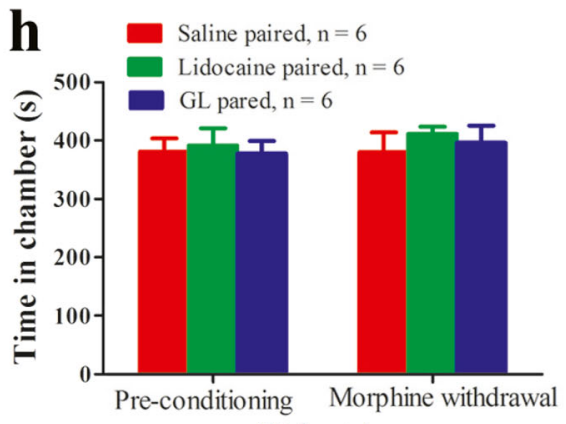

(Male rats)
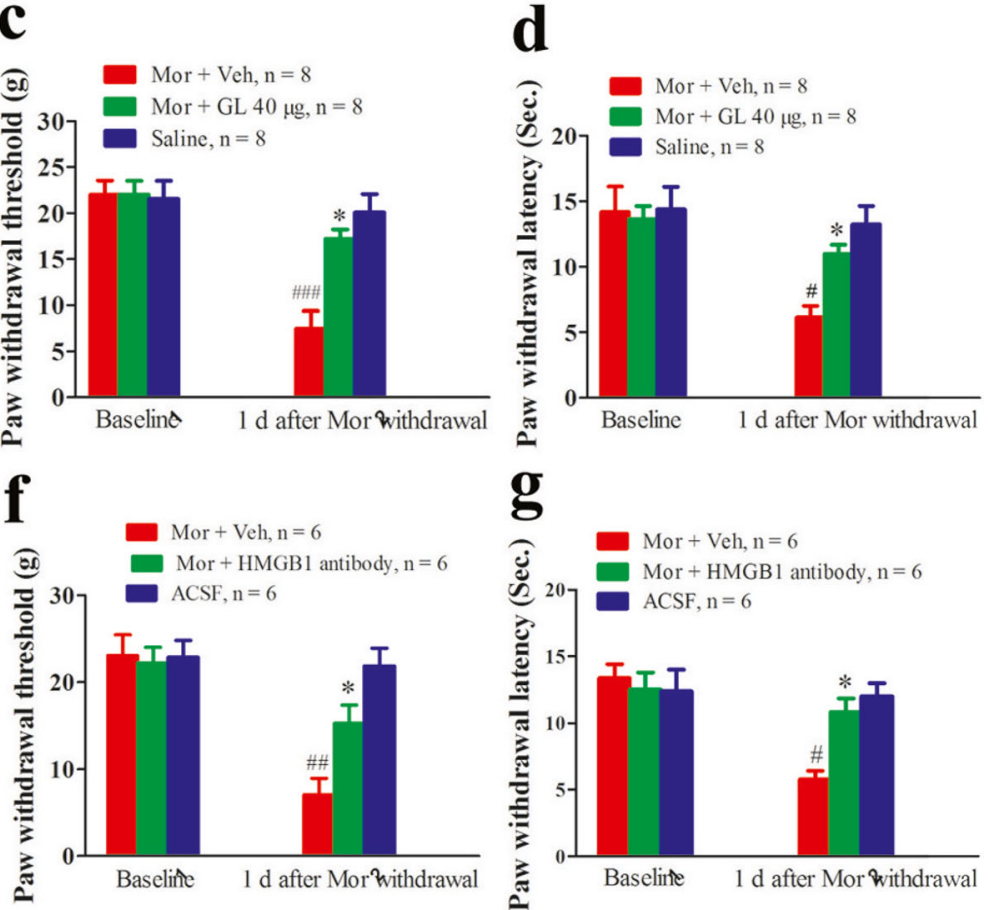

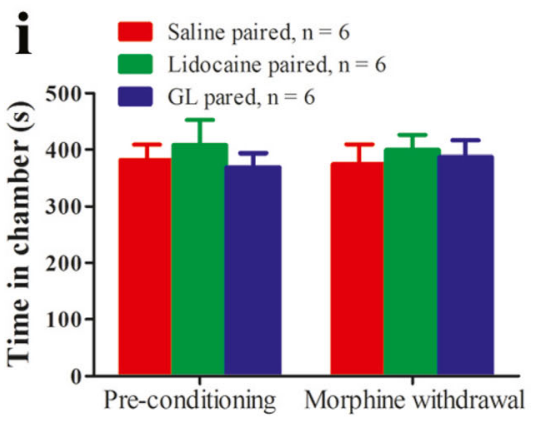

(Female rats)
Fig. 6 The role of spinal HMGB1 in morphine tolerance and morphine withdrawal-induced hyperalgesia in female rats. (a) Repeated intrathecal (i.t.) injections of morphine led to a significant increase in the expression of HMGB1, TLR4, and RAGE proteins in the spinal dorsal horn. $* P<$ 0.05 , $* * P<0.01$ vs. control group (i.t. injection of saline daily for 6 days). (b) Intrathecal coadministration of morphine (Mor) plus glycyrrhizin (GL), an inhibitor of HMGB1, prevented the decrease in the MPAE. $* P<0.05$; ** $P<0.01$; *** $P<0.001$ vs. morphine plus vehicle (Veh) group (one-way ANOVA). (c, d) Repeated intrathecal coinjections of morphine plus GL prevented the reduction in PWT and PWL after morphine withdrawal. * $P<0.05$; ** $P<0.01$ vs. morphine plus vehicle group (Student's $t$-test). (e) The reduction in the MPAE

induced by repeated i.t. injections of morphine were also partially reversed by the i.t. coinjection of morphine with an HMGB1 neutralizing antibody starting at day 7. * $P<0.05, * * P<0.01$ vs. morphine plus vehicle group (Student's $t$-test). (f, g) Coadministration of morphine with an HMGB1 neutralizing antibody alleviated morphine withdrawalinduced mechanical allodynia (f) and thermal hyperalgesia (g). $* P<$ 0.05 vs. morphine plus vehicle group (Student's $t$-test). (h, i) All rats, male (h) and female (i) showed equivalent time in the pairing chambers prior to conditioning day. After rats received vehicle, clonidine, and GL i.t. injection, the times that spent in chambers between vehicle-, clonidine, and GL-paired group were no reached statistical difference at testing day in male(h) and female rats (i). 
$6 \mathrm{~h}, \mathrm{i})$. It implies that morphine withdrawal did not lead to spontaneous pain in male and female rats.

\section{The signaling pathway involved in the morphine-mediated increased expression and release of HMGB1 in cultured spinal neurons and in spinal cord}

Previous studies have revealed that not only opioid receptors but also TLR4 likely function as receptors of morphine in the spinal cord [5]. To investigate the signaling pathway of morphine-induced HMGB1 expression and release, an in vitro study was performed with primary spinal neuron culture. CTOP, a specific antagonist of $\mu$ opioid receptors; TAK-242, a specific antagonist of TLR4; and naloxone $(50 \mu \mathrm{g})$, a nonspecific opioid receptor antagonist that has also been demonstrated to act as a TLR4 antagonist were added to media alone or in combination with morphine. Compared with control treatment (saline plus vehicle), morphine plus vehicle treatment led to a markedly increased expression in HMGB1 in cultured spinal neurons $(P<0.05)$. This increase was not prevented in the morphine plus CTOP group $(P>0.05$, Fig. 7b), whereas it was clearly inhibited when the cells were treated with morphine plus TAK-242 (morphine plus vehicle vs. morphine plus TAK-242: $P<0.05$, Fig. 7c) or morphine with naloxone (morphine plus vehicle vs. morphine plus naloxone: $P<0.05$, Fig. $7 \mathrm{~d}$ ). The basal expression levels of HMGB1 were not changed by treatment with CTOP, TAK-242, or naloxone alone. To further confirm these pharmacological observations, TLR4 siRNA was used to knockdown the expression of TLR4 in the cultured spinal neurons. In the morphine plus TLR4 siRNA group, but not in the scrambled RNA group, the expression of TLR4 decreased $121.23 \%$ compared to that in the morphine plus vehicle group $(P<0.05$, Fig. 7 e) . The morphine-induced increased expression of HMGB1 was significantly inhibited in the morphine plus TLR4 siRNA group $(P<0.05$ vs. morphine plus vehicle group, Fig. 7e). Similar to the effect of CTOP treatment on morphine-induced HMGB1 expression, the HMGB1 levels in media were not changed significantly in the group receiving morphine with CTOP $(P>0.05$ vs. morphine plus vehicle group, Fig. 7f). However, the release of HMGB1 from cultured neurons treated with morphine with TAK-242 $(P<0.01$ vs. morphine plus vehicle group, Fig. $7 \mathrm{~g})$, morphine with naloxone $(P<0.01$ vs. morphine plus vehicle group, Fig. 7h), and morphine with TLR4 siRNA $(P<0.05$ vs. morphine plus vehicle group, Fig. 7i) decreased significantly. The release of HMGB1 in the vehicle alone-treated group were not changed compared to that in the normal control group (in which nothing was added to the media), in the above 4 experiments
(Fig. 7f-i). To assess the quantity of HMGB1 released from dead cells, the neuronal death assay was performed by double staining cultured neurons with propidium iodide (PI, red staining, to label dead cells) and Hoechst 33342 (to label all cells). The result showed that the death rate between the normal and morphine-treated cells was not changed significantly $(0 \mu \mathrm{M}$ morphine vs. $20 \mu \mathrm{M}$ morphine: $12.31 \pm 3.69 \%$ vs. $13.78 \pm 4.17 \%, P>0.05$, Fig. $7 \mathrm{j}-\mathrm{m})$. This implies that the morphine-induced increase in expression and release of HMGB1 in cultured spinal neurons might be mediated by TLR4 signaling.

Then, an in vivo study was carried out to further validate the role of TLR4 signaling in morphine-mediated spinal HMGB1 expression through the coadministration of morphine with TAK-242 intrathecally. Compared with injections of the control treatment (saline plus vehicle), i.t. injections of morphine plus vehicle resulted in a significant increase in the expression of p-p65 in the dorsal horn of the lumbar enlargement segments (Fig. 8a). This increase was clearly inhibited by the coinjection of morphine with TAK242 (20 $\mu$ g once daily for 3 days) $(P<0.01$, Fig. 8 a). In addition, the intrathecal morphine-induced enhanced expression of HMGB1 in the spinal cord was markedly inhibited by the coinjection of morphine with TAk-242 (morphine plus vehicle vs. morphine plus TAK-242: $P<$ 0.05 , Fig. 8a). To further confirm that TLR4/NF-KB signaling activation mediates HMGB1 expression, repeated i.t. injections of morphine with PDTC $(0.5 \mu \mathrm{g}$ once daily for 3 days), an inhibitor of NF-KB activation, was performed. The results showed that the increased expression of HMGB1 in the morphine plus vehicle group was significantly inhibited by i.t. injections of PDTC (morphine plus vehicle vs. morphine plus PDTC: $P<0.05$, Fig. 8 b). As reported by a previous study, repeated i.t. injections of TAK-242 prevented the reduction of morphine's MPAE (morphine plus TAK-242 vs. morphine plus vehicle: $* P$ $<0.05$, ** $P<0.01$, Fig. 8c) and alleviated morphine withdrawal-induced mechanical allodynia and thermal hyperalgesia (morphine plus TAK-242 vs. morphine plus vehicle: $* P<0.05$, $* * P<0.01$, Fig. $8 \mathrm{~d}$ and c). These results indicate that TLR4/NF- $\mathrm{KB}$ signaling activation mediates the increase in HMGB1 expression in the spinal cord following repeated i.t. injections of morphine.

\section{The molecular mechanisms of spinal HMGB1-mediated morphine analgesic tolerance and hyperalgesia}

To investigate the mechanisms of HMGB1-mediated morphine tolerance, the expression levels of NF- $\mathrm{kB}$ p-p65, IKB- $\alpha, p-J N K$, and $p-p 38$ in the dorsal horn of the lumbar enlargement were examined following repeated i.t. injection of HMGB1 siRNA with morphine. Compared with 

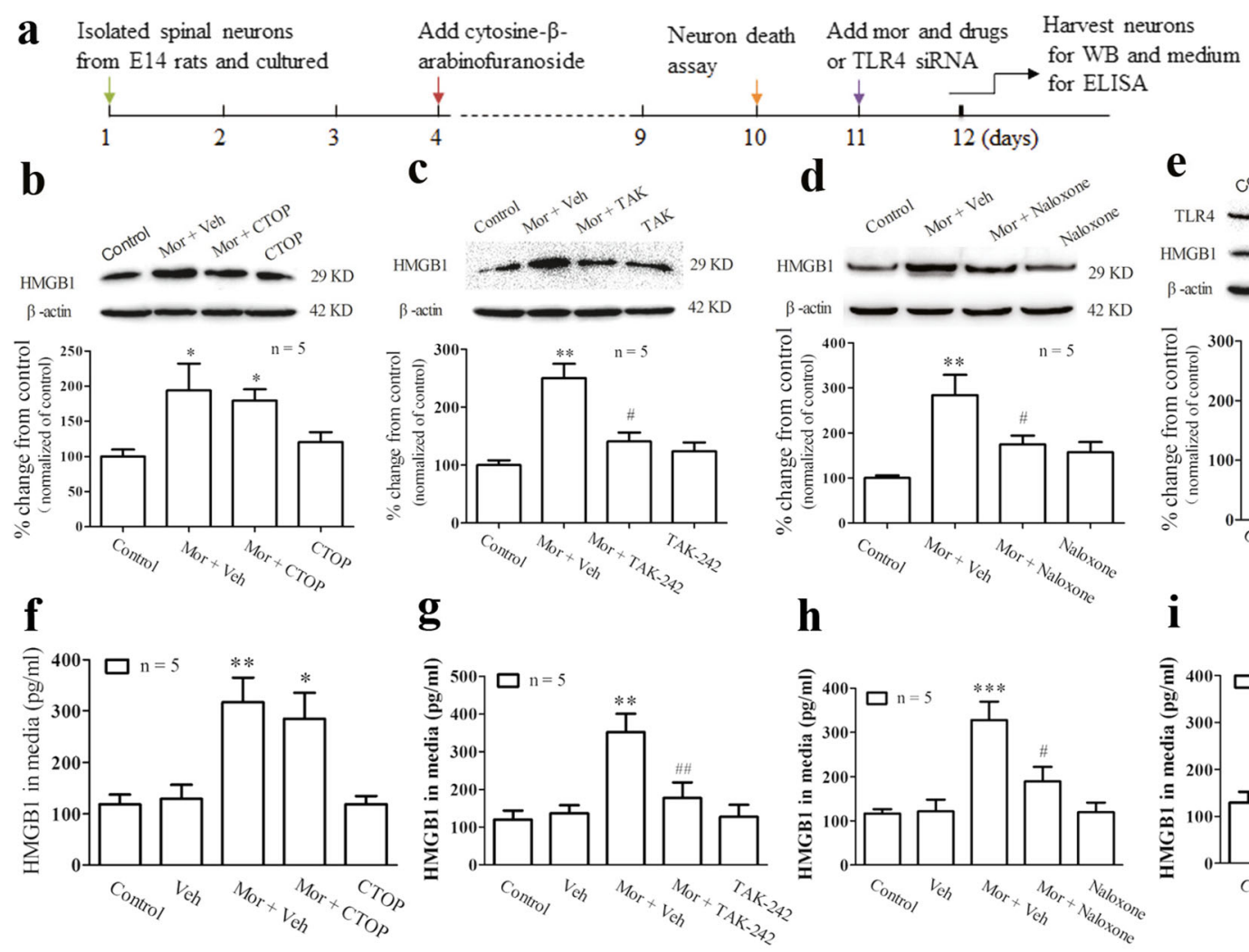

d
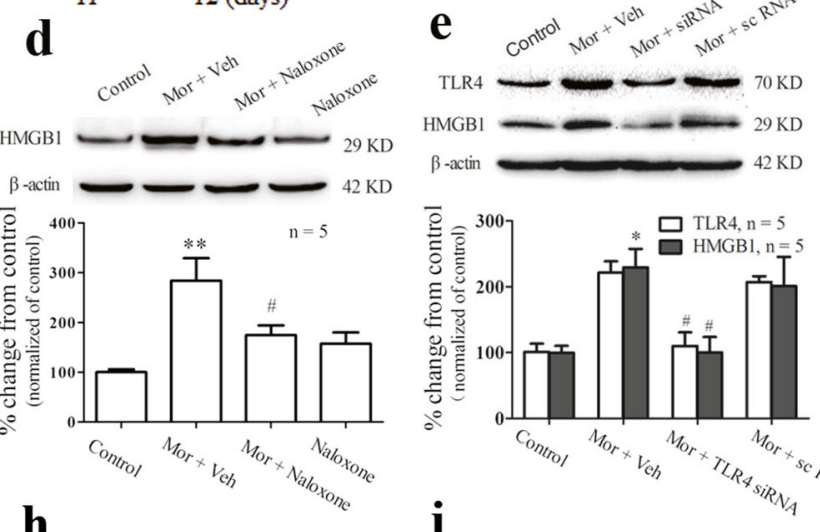

h
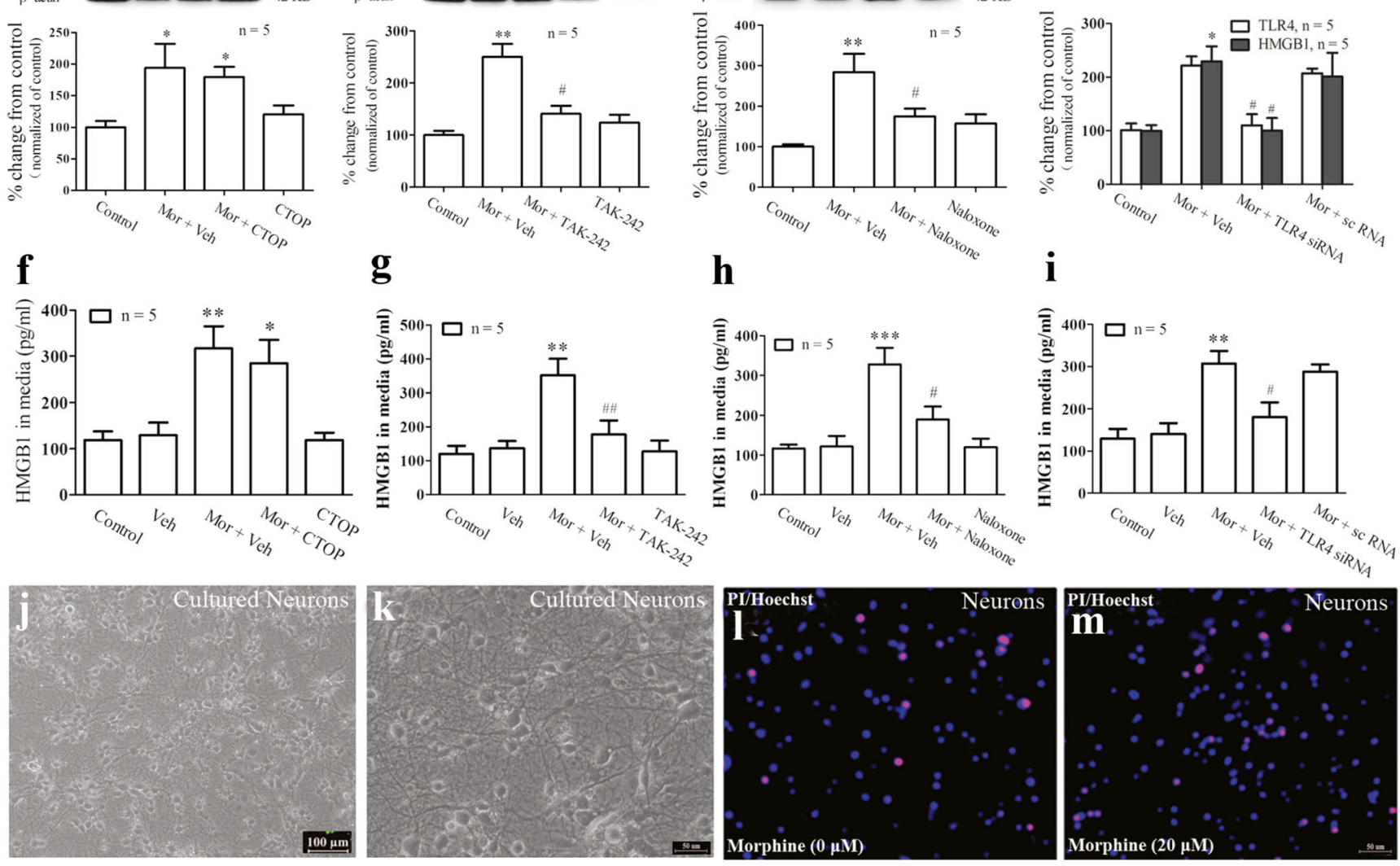

significant reductions in the release of HMGB1 from cultured neurons. Compared to the normal control neurons (in which nothing was added to the media), the neurons treated with vehicle alone did not exhibit a change in the levels of HMGB1 released. $* P<0.05$, ** $P<0.01$ vs. control (Vehicle). \# $P<0.05$, \#\# $P<0.01$ vs. morphine plus vehicle (Veh: b, c, d, $\mathbf{f}, \mathbf{g}$, and $\mathbf{h}$ : normal saline containing 10\% DMSO; e and i: transfection regent). Data are presented as the mean \pm SEM and were analyzed by oneway ANOVA. (j-m) Images showing the results of the cell death assay for cultured spinal neurons. (j) and (k) are the normal cultured neurons under different magnifications. (I) and (m) are the neurons treated with morphine and costained with Hoechst/propidium iodide (PI). Images in $\mathbf{j}$ scale bar $=100 \mu \mathrm{m} ; \mathbf{k}-\mathbf{m}$ scale bar $=50 \mu \mathrm{m}$. morphine plus vehicle treatment, coadministration of morphine with HMGB1 siRNA resulted in a significant decrease in the expression of HMGB1 $(P<0.01)$ and p-p65 $(P<0.01)$ and increased cytoplasmic IкB- $\alpha(P<0.05)$. However, levels of p-p38 $(P>0.05)$ and p-JNK $(P>0.05$, Fig. 9a) were not changed by the treatment. Morphine plus scramble RNA did not affect the increase in the expression of spinal HMGB1 ( $P<0.05$ vs. vehicle group) and p-p65 (** $P<0.01$ vs. vehicle group) or the decrease in the expression of IKB- $\alpha$ ( $* P<0.05$ vs. vehicle group, Fig. 9a). The total levels of NF-kB p65, JNK, and p38 were not changed in the morphine plus vehicle, morphine plus HMGB1 siRNA, or morphine plus HMGB1 scramble RNA groups compared to those in the vehicle group (data not shown). This finding indicates that spinal NF- $\mathrm{KB}$ signaling activation might mediate HMGB1 activity following chronic i.t. morphine exposure. To further confirm these results, i.t. injection of recombinant HMGB1 in 


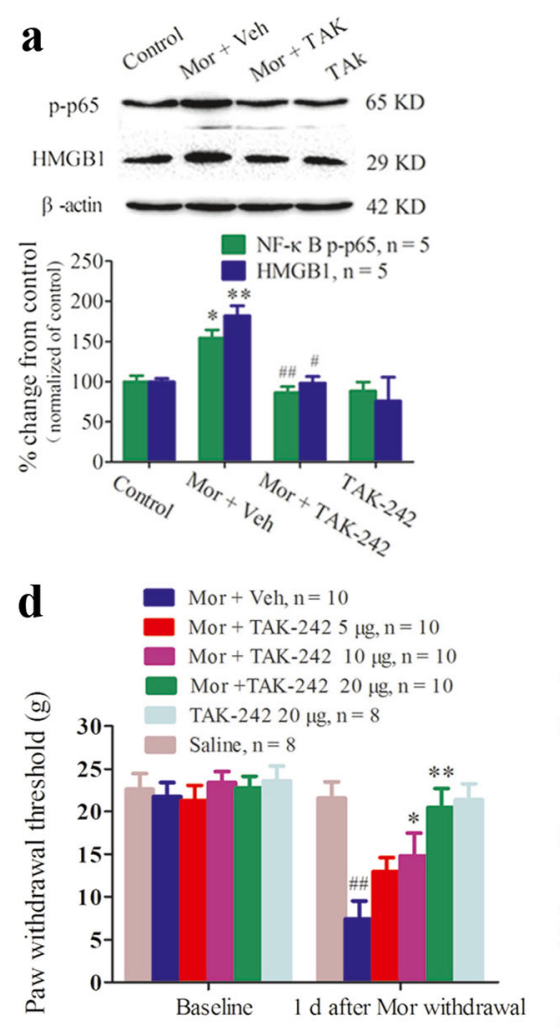

b
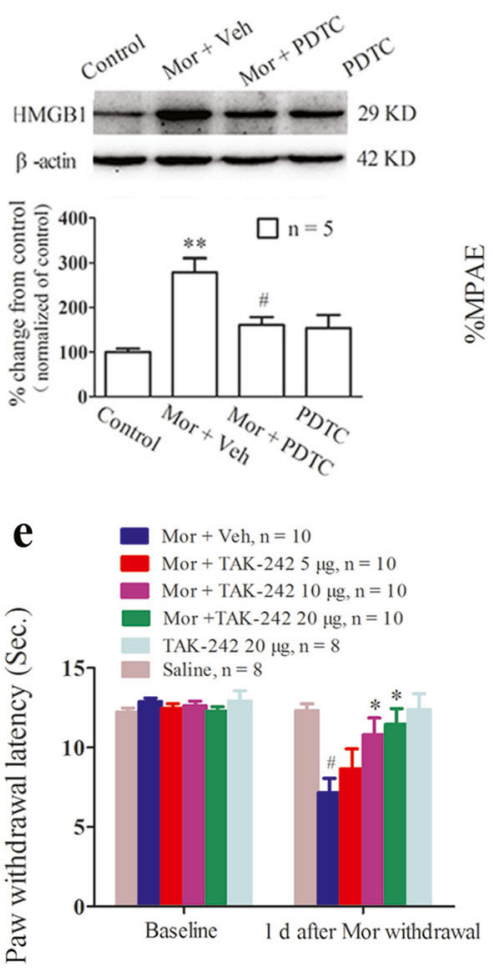

Fig. 8 Effects of TLR4/NF- $\mathrm{KB}$ signaling activation on the expression of HMGB1 in the spinal cord and the development of morphine tolerance and hyperalgesia. (a) Repeated i.t. coinjections of morphine with TAK242 inhibited the phosphorylation of NF-kB p65 and reduced HMGB1 expression. (b) Repeated i.t. coinjections of morphine with PDTC reduced HMGB1 expression. Data shown in (a) and (b) are presented as the mean \pm SEM and were analyzed by one-way ANOVA. $* P<0.05$, ** $P<0.01$ vs. control (i.t. saline containing $10 \%$ DMSO); \# $P<0.05$, \#\# $P$ $<0.01$ vs. morphine plus vehicle group. (c) Repeated i.t. coinjections of morphine plus TAK-242 dose-dependently prevented the decrease in morphine's MPAE. \#\#\# $P<0.001$ vs. day one (two-way ANOVA); ${ }^{*} P$
C

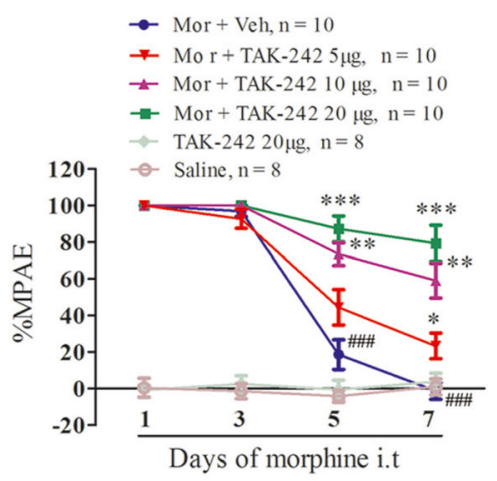

$<0.05, * * P<0.01, * * * P<0.001$ vs. morphine plus vehicle group (oneway ANOVA). Repeated i.t. injections of saline or TAK-242 alone did not change the basal MPAE value. (d, e) Chronic i.t. morphine exposure resulted in decreased paw withdrawal threshold (PWT) (d) and paw withdrawal latency (PWL) (e) in the left hind paw. \#\# $P<0.01$ vs. baseline. Repeated i.t. coinjections of morphine plus TAK-242 prevented the reduction in PWT and PWL after morphine withdrawal. $* P<0.05$; $* * P<$ 0.01 vs. morphine plus vehicle group (Student's $t$-test). The basal tailflick response, PWT and PWL were not changed by repeated i.t. injections of TAK-242 alone. naïve rats was performed. Compared with the control treatment (i.t. injection of ACSF), bolus HMGB1 i.t. injection caused significantly increased expression of p-p65 starting at 3 hours $(P<0.05)$ and persisting to 6 hours $(P$ $<0.05$, Fig. 9 b) after injection into the spinal cord. Spinal TNF- $\alpha$ and IL-1 $\beta$ expression was also upregulated 3 hours after HMGB1 administration $(* P<0.05$, ** $P<$ 0.01 vs. control, Fig. 9b). However, these increased expression levels were markedly inhibited by coinjection of TAK-242 (\# $P<0.05$ vs. HMGB1 plus vehicle, Fig. 9c). Behavioral observations showed that the coinjection of morphine with HMGB1 led to significant reductions in the MPAE $(* P<0.05$ vs. morphine plus vehicle, Fig. $9 \mathrm{~d})$, which occurred one hour after the treatment. The PWTs and PWLs in the hind paw were also decreased in the animals receiving i.t. injection of HMGB1 $(* P<0.05$ vs. ACSF i.t., Fig. 9e and f), and this change occurred at 3 hours and persisted to 6 hours after the treatment. Taken together, these results imply that TLR4-NF- $\mathrm{kB}$ signaling activation through the upregulation of TNF- $\alpha$ and IL-1 $\beta$ in the spinal dorsal horn may mediate HMGB1 activity during the development of morphine tolerance and hyperalgesia.

\section{Discussion}

Here, we studied the role of spinal HMGB1 in the pathogenesis of morphine tolerance and hyperalgesia as well as its underlying mechanisms. Our results showed that chronic morphine exposure resulted in an increase in the expression of HMGB1 and TLR4 in spinal neurons, astrocytes, and microglia. And these upregulations contribute to the development and maintenance of morphine tolerance and hyperalgesia via TLR4/NF-KB signaling 

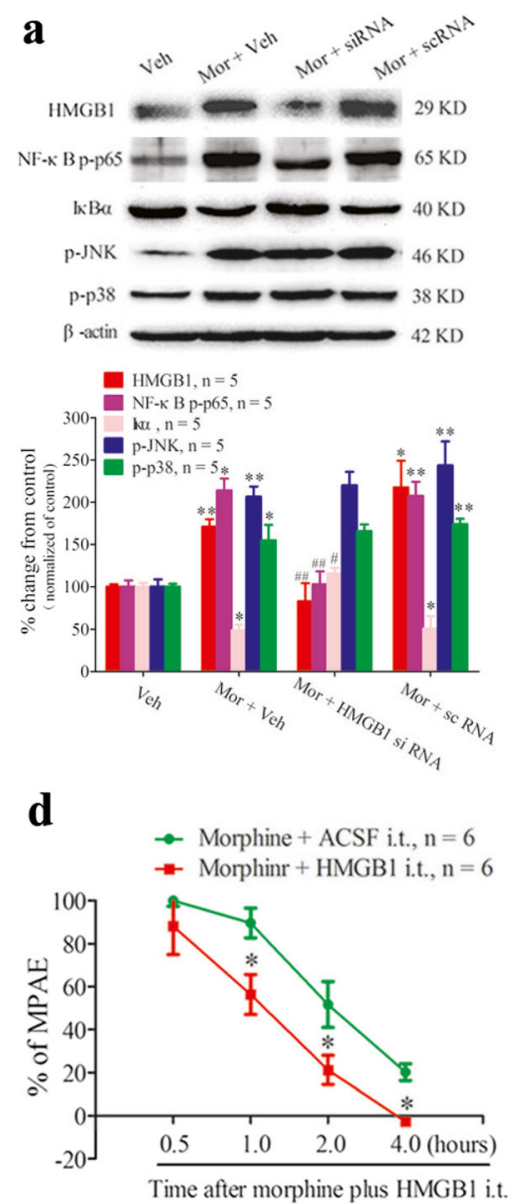
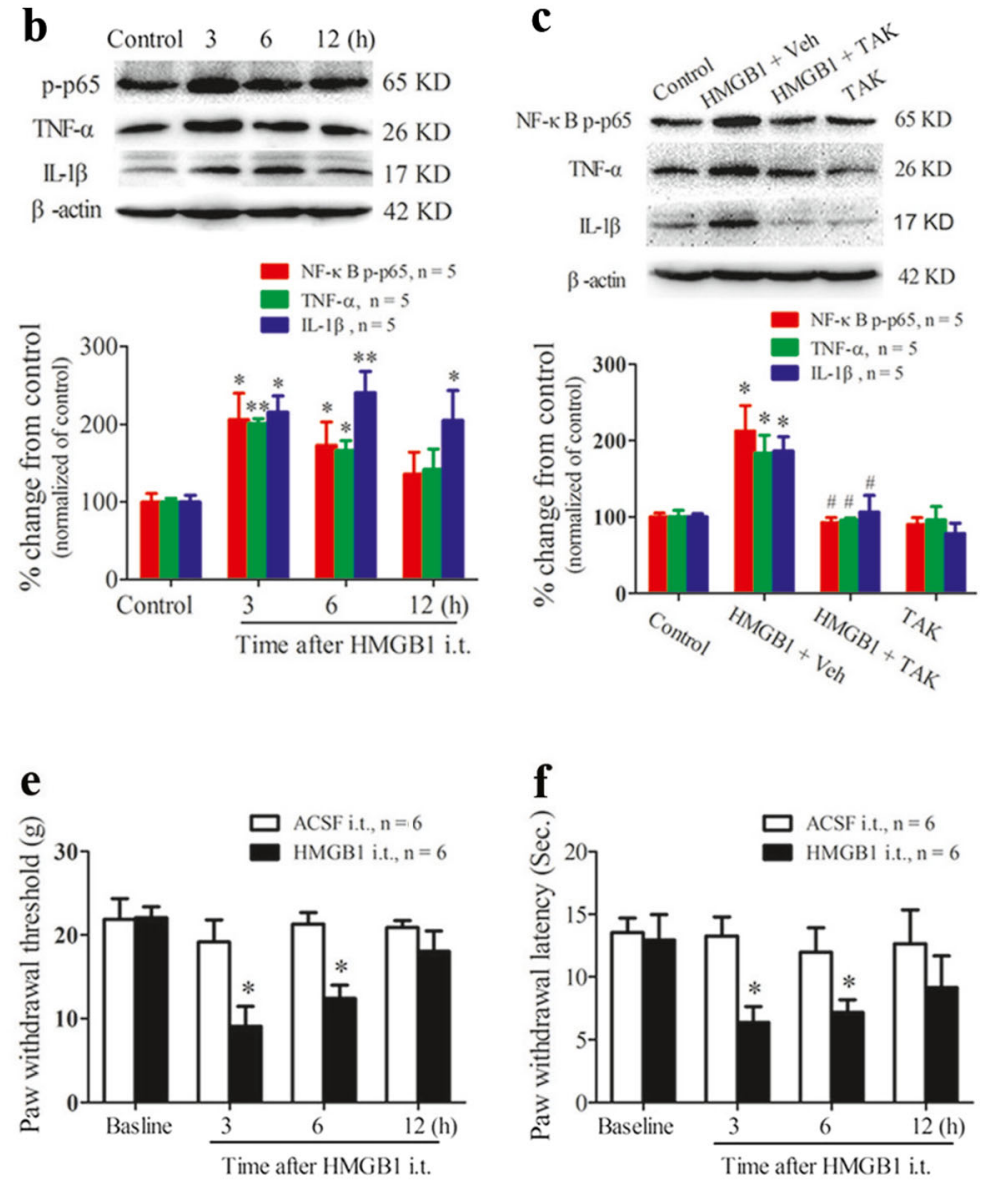

Fig. 9 Signaling pathway of spinal HMGB1-mediated morphine tolerance and hyperalgesia. (a) Compared to morphine plus vehicle treatment, intrathecal administration of morphine with HMGB1 siRNA inhibited the increase in NF- $\mathrm{BB}$ p-p65 levels and the decrease in cytoplasmic IкB- $\alpha$ levels. However, p-p38 and p-JNK levels were not changed by this treatment. Data are presented as the mean \pm SEM and were analyzed by oneway ANOVA. $* P<0.05$, ** $P<0.01$ vs. vehicle (Veh: transfection regent); \# $P<0.05$, \#\# $P<0.01$ vs. morphine plus vehicle or morphine plus scramble (sc) RNA group. (b, c) A single i.t. injection of recombinant HMGB1 resulted in the increased expression of p-p65, TNF- $\alpha$ and IL-1 $\beta$ in the dorsal horn at 3 hours, which persisted to 6 hours after the

mediated releasing of pro-inflammatory cytokines IL-1 $\beta$ and TNF- $\alpha$.

\section{Chronic morphine exposure increases the expression of HMGB1 in the spinal dorsal horn}

Compelling evidence has demonstrated that chronic morphine exposure induces sterile neuroinflammation in the spinal cord, which has been linked to the suppression of morphine analgesia as well as the enhancement of morphine tolerance and hyperalgesia $[5,39,40]$. HMGB1 has been implicated as a key factor in the mediation of neuroinflammatory processes in several pathophysiological conditions, including seizure, ischemia, and chronic pain $[9,11,41]$. However, whether chronic morphine exposure can lead to the expression and injection (b). These effects were partially blocked by the coinjection of HMGB1 with TAK-242 (c). $* P<0.05$, $* * P<0.01$ vs. control (i.t. ACSF); \# $P<0.05$ vs. HMGB1 plus vehicle (Veh: ACSF). (d) A bolus i.t. coinjection of morphine with HMGB1 reduced the analgesic efficacy of morphine. $* P<0.05$ vs. morphine plus ACSF group. (e-f) Bolus i.t. injection of HMGB1 led to a reduction in the paw withdrawal threshold (e) and paw withdrawal latency (f) in the left hind paw, which occurred at 3 hours and persisted to 6 hours after the injection. $* P<0.05$ vs. baseline; $\# P<0.05$ vs. ACSF group. ACSF: artificial cerebrospinal fluid. Data of (d), (e), and (f) were analyzed by Student's $t$-test.

release of HMGB1 in the nervous system remains unclear. In the current study, we found that chronic i.t. morphine injection increased the expression of HMGB1 in spinal dorsal horn neurons, astrocytes, and microglia. qPCR results also showed significantly increased expression of spinal HMGB1 mRNA following chronic i.t. morphine administration. Results from female rats also showed clear increased repression expression of HMGB1 in the spinal cord after repeated i.t. injections of morphine. To observe the systemic effect of morphine and to exclude the possible effect of i.t. injection itself on HMGB1 expression, an experiment of repeated s.c. injections of morphine in mice was performed, and the result showed an significant increased expression of HMGB1 mRNA in the dorsal horn. It implies that morphine works as a potent inducer of HMGB1 expression in spinal cord. It has 
been reported that HMGB1 can be detected in the cell culture media and cytosol of BV2 cells, a microglial cell line, in response to LPS stimulation and in human astrocytes in response to IL-1 $[8,42]$. In the present study, to obtain more direct evidence of morphine-induced HMGB1 expression and release, primary spinal neuron culture was used. We found that morphine challenge led to the enhanced expression of HMGB1 in cultured neurons, which correlated with an increase in the levels of the HMGB1 protein in the culture media. These results indicating that morphine treatment not only promote HMGB1 expression, but also stimulate it release. Our in vivo double immunofluorescence staining and a previous study [43], in which a model of chronic pain was used, showed that in addition to neurons, spinal astrocytes and microglia also displayed significantly increased expression of HMGB1. Therefore, although the present study showed a direct effect of morphine on cultured neurons on HMGB1 release, in vivo conditions, the main source of HMGB1 might be its release from activated glial cells following morphine exposure.

\section{Signaling pathway of morphine-induced spinal HMGB1 expression and release}

It has been well documented that HMGB1 release occurs either passively or actively during pathogen invasion and tissue injury. The passive release of HMGB1 from dead, dying or injured cells is rapid and associated with increased cell permeability, whereas active secretion occurs more slowly and is initiated by membrane receptor interactions with extracellular products and intracellular signal transduction in which TLR4 plays a critical role $[9,11]$. Here, we found that the morphineinduced increased expression and release of HMGB1 in cultured spinal neurons was suppressed by TAK-242, naloxone, and TLR4 siRNA, but not by CTOP, a specific antagonist of $\mu$-opioid receptors. Both TAK-242 and naloxone have been identified as antagonists of TLR4 [44]. By double immunofluorescence staining and Hoechst/propidium iodide costaining of cultured cells, we found that the neuron purity reached $88.7 \%$ and the cell death rate was less than $14 \%$. Using a method of fluorescence in situ hybridization combined with immunofluorescence staining, we found that both the TLR4 protein and mRNA colocalized with the neuronal marker NeuN in cultured spinal neurons. Therefore, the source of HMGB1 in media was likely TLR4-mediated active release from cultured neurons following morphine exposure. Our in vivo study indicated that repeated intrathecal coinjections of morphine with TAK-242 also resulted in the reduced expression of HMGB1, which correlated with inhibited NF-KB activation in the dorsal horn. Double immunofluorescence staining revealed that both TLR4 and HMGB1 colocalized with spinal neurons, astrocytes, and microglia. Token together these in vitro and in vivo results have provided clear evidence and indicate that TLR4/ NF- $\mathrm{KB}$ signaling activation mediates the increase in HMGB1 expression and its release in the spinal cord following chronic morphine exposure. Hutchinson et al. [15] reported that repeated i.t. coinjections of morphine with TAK-242 prevents the reduction of morphine's MPAE and alleviates morphine withdrawal-induced hyperalgesia. However, previous studies have reported contradictory findings regarding the cell types that express TLR4 in the nervous system. Some researchers have reported that TLR4 is expressed in neurons and satellite glial cells in the DRG and is highly expressed in microglia, somewhat in astrocytes, but not in neurons in the central nervous system [45]. Liu et al. [46] reported that in addition to astrocytes displaying robust expression of TLR4, spinal neurons are also positively stained in a chronic itch model. In the brain, the HMGB1-TLR4 pathway is upregulated in the neurons and astrocytes inside focal cortical dysplasia type II lesions in children [47] as well as in short- and long-term cultures of rat hippocampal neurons [48]. We think these discrepancies may be due to the different models and time points used. Although our in vitro study showed a direct effect of morphine-mediated HMGB1 release from cultured spinal neurons, the main source of HMGB1 in vivo may be spinal glial cells, which are mediated by TLR4/NF-KB signaling activation.

\section{Spinal HMGB1 level upregulation is involved in the development and maintenance of morphine tolerance and hyperalgesia}

Although the role of HMGB1 in chronic pain has been implicated by previous studies [41], the role of HMGB1 in morphine tolerance and morphine withdrawal-induced hyperalgesia still remains elusive. Our present study in male and female rats found that chronic i.t. morphine exposure results in the upregulation of HMGB1 in the spinal cord and the reduction of morphine's MPAE at day 7 of repeated i.t. injections of morphine. Morphine withdrawal induced clear mechanical allodynia and thermal hyperalgesia, but no spontaneous pain when examined by CPP. Spinal HMGB1 knockdown or i.t. injection of GL, an inhibitor that can bind directly to HMGB1 and inhibit its extracellular cytokine activities [20, $21,38]$, effectively prevented the reduction in morphine's MPAE and alleviated mechanical allodynia and thermal hyperalgesia after morphine withdrawal. Established morphine tolerance was also partially reversed by i.t. injections of an HMGB1 neutralizing antibody and GL. Because chronic i.t. morphine exposure also increased the expression of HMGB1 in L4 and L5 DRGs, it cannot be excluded that the effect of intrathecal HMGB1 siRNA, GL, and an HMGB1 neutralizing antibody on morphine tolerance involved the DRG. These results imply that morphineinduced upregulation of HMGB1 in spinal cord was involved in the development and maintenance of morphine tolerance 
and morphine withdrawal-induced hyperalgesia. Recently, Grace et al. [49] reported that a short course of morphine treatment starting 10 days after chronic sciatica nerve injury results in increased spinal HMGB1 release via TLR4 and $\mathrm{P} 2 \mathrm{X} 3 \mathrm{R}$ that is responsible for maintaining persistent allodynia after morphine ceases. This is the only published paper that has observed this effect of morphine treatment on spinal HMGB1 expression in established neuropathic pain. Their results support our present finding that morphine-mediated HMGB1 expression is involved in morphine tolerance and spinal pain processing.

\section{Downstream molecular mechanisms of spinal HMGB1-mediated morphine tolerance}

Previous studies have revealed that HMGB1 mediates its activities by serving as a ligand for several different receptors, including TLR2, TLR4, TLR5, TLR9, and RAGE [9]. HMGB1 mediates the chemotaxis, proliferation, and differentiation of immune cells and other cells through RAGE signaling. Additionally, HMGB1-mediated TLR4 signaling is a strong inducer of cytokine production in a fashion strictly dependent on the redox state of HMGB1 [11, 12]. In a paradigm of 6-day i.t. injections of morphine, we detected markedly increased expression of TLR4 and RAGE in the spinal dorsal horn. This finding is in agreement with previous studies [39, 50]. However, the mechanisms of morphine-induced upregulation of TLR4 in central nervous system (CNS) still remain elusive. It has been reported morphine exposure results in a downregulated TLR4 expression via $\mu$-opioid receptor in cultured murine macrophages [51]. But, a $\mu$-opioid receptor-independent increased expression of TLR4 is evidenced in the spinal cord and brain following chronic morphine exposure [52]. It suggests that may have different mechanisms of morphineinduced TLR4 expression between peripheral and CNS, and its detail signal pathway still need to further study in future. We also found that spinal HMGB1 knockdown by i.t. injection of HMGB1 siRNA clearly reduced the phosphorylation level of NF- $k$ B p 65 and increased cytoplasmic IkB- $\alpha$ (an inhibitor of NF- $k B$ p65 translocation to the nucleus and degradation after dissociation from p65) but did not influence the activation of MAPK p38 and JNK in the spinal cord following chronic i.t. morphine exposure. It indicates that morphine-induced TLR4/ NF- $\mathrm{KB}$ signaling activation dependent on the effect of spinal HMGB1. To further verify the signal pathway and the effect of spinal HMGB1 on morphine's analgesic efficacy, a bolus i.t. injection of recombinant disulfide HMGB1 in naïve rats was performed because only disulfide HMGB1 combines with TLR4 and exhibits proinflammatory cytokine activity [53,54]. Our results showed that a single dose of HMGB1 i.t. injection caused a significant reduction in morphine's MPAE and painrelated hypersensitivity in the hind paws. Moreover, the expression levels of phosphorylated NF-KB p65, TNF- $\alpha$, and IL-1 $\beta$ in the spinal cord were also significantly increased after HMGB1 i.t. injection and blocked by coinjection of TAK242. These results imply that spinal HMGB1 is sufficient to lead to spinal neuroinflammation and reduce the analgesic efficacy of morphine and that the effects are dependent on TLR4/ NF- $\kappa$ B signaling activation. Our results also imply that may have a positive loop which started from morphine-mediated TLR4 activation, ended at increased expression and release of HMGB1, IL- $1 \beta$, and TNF- $\alpha$, and backed by the HMGB1 to cause stronger TLR4/NF- $\mathrm{KB}$ signaling activation and more secretions of HMGB1, IL- $1 \beta$, and TNF- $\alpha$ in spinal cord, and at last decreased the analgesic effect of morphine by aggravating the local neuroinflammation. Therefore, the inhibition of HMGB1 release and activity might be a promising therapeutic strategy to prevent morphine tolerance in the clinic. Moreover, our present study also observed significantly increased expression of RAGE mRNA and protein in the spinal cord following repeated i.t. injection of morphine. Hence the response of RAGE to chronic i.t. morphine exposure needs to be verified in the future.

\section{Conclusions}

The present study have provided evidence, for the first time in our knowledge, that inhibition of spinal HMGB1 activity effectively prevented the reduction of analgesic tolerance, and alleviated morphine withdrawal-induced hyperalgesia. The underlying mechanisms involves in the inhibition of HMGB1-mediated IL-1 $\beta$ and TNF- $\alpha$ release. Therefore, the HMGB1 inhibitor might be a promising adjuvant to morphine in treatment of intractable pain in the clinic, which it may not only prevent the development of morphine tolerance, but also reduce its side effects.

Acknowledgements This work was supported by grants from the National Natural Science Foundation of China (81571079 and 81501070).

Required Author Forms Disclosure forms provided by the authors are available with the online version of this article.

Authors' Contributions J.T. X. conceived the project and supervised all experiments. J.T.X., J.Q., Y. Z., and Y.X.T. designed the project. J.Q., Y. Z., and J.Z. produced the animal model, conducted the behavioral experiments, and carried out the Western blot assays and siRNA transfection. Y.G., M.Y., W.Z., and H.G. performed the PCR and immunohistochemistry experiments. L.B., M.J., and F.X., carried out the cell culture, ELISA, and fluorescence in situ hybridization experiments. J.Q., Y.Z., Y.G., and J.T.X. analyzed the data. J.T.X. wrote and Y.X. T. revised the manuscript. All of the authors read and discussed the manuscript.

\section{Compliance with Ethical Standards}

All procedures used were approved by the Animal Care and Use Committee at Zhengzhou University, PR China. 
Competing Interests The authors declare that they have no conflict of interest.

\section{References}

1. Sommer C. Peripheral neuropathies: Long-term opioid therapy in neuropathy: benefit or harm? Nat Rev Neurol 2017; 13: 516-517.

2. Martyn JAJ, Mao J, Bittner EA. Opioid tolerance in critical illness. N Engl J Med 2019; 380:365-378.

3. Mayer DJ, Mao J, Holt J, Price DD. Cellular mechanisms of neuropathic pain, morphine tolerance, and their interactions. Proc Natl Acad Sci U S A 1999; 96: 7731-7736.

4. Roeckel LA, Le Coz GM, Gaveriaux-Ruff C, Simonin F. Opioidinduced hyperalgesia: Cellular and molecular mechanisms. Neuroscience 2016; 338: 160-182.

5. Grace PM, Maier SF, Watkins LR. Opioid-induced central immune signaling: implications for opioid analgesia. Headache 2015; 55: 475-489.

6. Pasternak GW. Mu Opioid Pharmacology: 40 Years to the Promised Land. Adv Pharmacol 2018; 82: 261-291.

7. Ueda T, Yoshida M. HMGB proteins and transcriptional regulation. Biochim Biophys Acta 2010; 1799: 114-118.

8. Andersson U, Wang H, Palmblad K, et al. High mobility group 1 protein (HMG-1) stimulates proinflammatory cytokine synthesis in human monocytes. J Exp Med 2000; 192: 565-570.

9. Andersson U, Tracey K J. HMGB1 is a therapeutic target for sterile inflammation and infection. Annu Rev Immunol 2011; 29: 139162.

10. Frank MG, Adhikary S, Sobesky JL, Weber MD, Watkins LR, Maier SF. The danger-associated molecular pattern HMGB1 mediates the neuroinflammatory effects of methamphetamine. Brain Behav Immun 2016; 51: 99-108.

11. Harris HE, Andersson U, Pisetsky DS. HMGB1: a multifunctional alarmin driving autoimmune and inflammatory disease. Nat Rev Rheumatol 2012; 8: 195-202.

12. Van B Jr, Buurman WA, Griffioen AW. Convergence and amplification of toll-like receptor (TLR) and receptor for advanced glycation end products (RAGE) signaling pathways via high mobility group B1 (HMGB1). Angiogenesis 2008; 11: 91-99.

13. Eidson LN, Murphy AZ. Blockade of Toll-like receptor 4 attenuates morphine tolerance and facilitates the pain relieving properties of morphine. J Neurosci 2013; 33: 15952-15963.

14. Hutchinson MR, Northcutt AL, Hiranita T, et al. Opioid activation of toll-like receptor 4 contributes to drug reinforcement. J Neurosci 2012; 32: 11187-11200.

15. Hutchinson MR, Zhang Y, Shridhar M, et al. Evidence that opioids may have toll-like receptor 4 and MD-2 effects. Brain Behav Immun 2010; 24: 83-95.

16. Lin CP, Lu DH. Role of Neuroinflammation in Opioid Tolerance: Translational Evidence from Human-to-Rodent Studies. Adv Exp Med Biol 2018;1099:125-139.

17. Hutchinson MR, Coats BD, Lewis SS, et al. Proinflammatory cytokines oppose opioid-induced acute and chronic analgesia. Brain Behav Immun 2008;22:1178-1189.

18. Storkson RV, Kjorsvik A, Tjolsen A, Hole K. Lumbar catheterization of the spinal subarachnoid space in the rat. J Neurosci Methods 1996; 65: 167-172.

19. Ma F, Kouzoukas DE, Meyer-Siegler KL, Westlund KN, Hunt DE, Vera PL. Disulfide high mobility group box-1 causes bladder pain through bladder Toll-like receptor 4. BMC Physiol 2017; 17:60032 .

20. Kim SW, Jin Y, Shin JH, et al. Glycyrrhizic acid affords robust neuroprotection in the postischemic brain via anti-inflammatory effect by inhibiting HMGB1 phosphorylation and secretion. Neurobiol Dis 2012; 46: 147-156.

21. Sun X, Zeng H, Wang Q, et al. Glycyrrhizin ameliorates inflammatory pain by inhibiting microglial activation-mediated inflammatory response via blockage of the HMGB1-TLR4-NF-kB pathway. Exp Cell Res 2018; 369, 112-119.

22. Xing F, Zhang W, Wen J, et al. TLR4/NF-kB signaling activation in plantar tissue and dorsal root ganglion involves in the development of postoperative pain. Mol Pain 2018;14:1744806918807050.

23. Bai L, Zhai C, Han K, et al. Toll-like receptor 4-mediated nuclear factor-kappaB activation in spinal cord contributes to chronic morphine-induced analgesic tolerance and hyperalgesia in rats. Neurosci Bull 2014;30:936-948

24. Hei Y, Chen R, Yi X, Long Q, Gao D, Liu W. HMGB1 Neutralization Attenuates Hippocampal Neuronal Death and Cognitive Impairment in Rats with Chronic Cerebral Hypoperfusion via Suppressing Inflammatory Responses and Oxidative Stress Neuroscience 2018;383:150-159.

25. Gong H, Su WJ, Cao ZY, et al. Hippocampal Mrp8/14 signaling plays a critical role in the manifestation of depressive-like behaviors in mice. J Neuroinflammation 2018;15:252-265.

26. Xu JT, Zhao JY, Zhao X, et al. Opioid receptor-triggered spinal mTORC1 activation contributes to morphine tolerance and hyperalgesia. J Clin Invest 2014;124:592-603.

27. Lim G, Wang S, Zeng Q, Sung B, Yang L, Mao J. Expression of spinal NMDA receptor and PKCgamma after chronic morphine is regulated by spinal glucocorticoid receptor. J Neurosci 2005;25: 11145-11154.

28. Chaplan SR, Bach FW, Pogrel JW, Chung JM, Yaksh TL. Quantitative assessment of tactile allodynia in the rat paw. J Neurosci Methods 1994;53:55-63

29. Hargreaves K, Dubner R, Brown F, Flores C, Joris J. A new and sensitive method for measuring thermal nociception in cutaneous hyperalgesia. Pain 1988;32:77-88.

30. King T, Vera-Portocarrero L, Gutierrez T, et al. Unkasking the tonic-aversive state in neuropathic pain. Nat Neurosci 2009;12: 1364-1366

31. Xing F, Kong C, Bai L, et al. CXCL12/CXCR4 signaling mediated ERK1/2 activation in spinal cord contributes to the pathogenesis of postsurgical pain in rats. Mol Pain 2017;13:1744806917718753

32. Ji RR, Samad TA, Jin SX, Schmoll R, Woolf CJ. p38 MAPK activation by NGF in primary sensory neurons after inflammation increases TRPV1 levels and maintains heat hyperalgesia. Neuron 2002;36:57-68.

33. Gu HW, Xing F, Jiang MJ, et al. Upregulation of matrix metalloproteinase- $9 / 2$ in the wounded tissue, dorsal root ganglia, and spinal cord is involved in the development of postoperative pain. Brain Res 2019;1718:64-74.

34. Schafers M, Geis C, Svensson CI, Luo ZD, Sommer C. Selective increase of tumor necrosis factor-alpha in injured and spared myelinated primary afferents after chronic constrictive injury of rat sciatic nerve. Eur J Neurosci 2003;17:791-804.

35. Zhao X, Tang Z, Zhang H, et al. A long noncoding RNA contributes to neuropathic pain by silencing Kcna2 in primary afferent neurons. Nat Neurosci 2013;16:1024-1031

36. Kurland DB, Gerzanich V, Karimy JK, et al. The Sur1-Trpm4 channel regulates NOS2 transcription in TLR4-activated microglia. J Neuroinflammation 2016;13:130-153

37. Bai L, Wang X, Li Z, et al. Upregulation of Chemokine CXCL12 in the Dorsal Root Ganglia and Spinal Cord Contributes to the Development and Maintenance of Neuropathic Pain Following Spared Nerve Injury in Rats. Neurosci Bull. 2016;32:27-40.

38. Ohnishi M, Katsuki H, Fukutomi C, et al. HMGB1 inhibitor glycyrrhizin attenuates intracerebral hemorrhage-induced injury in rats. Neuropharmacology 2011;61:975-980. 
39. Ellis A, Grace PM, Wieseler J, et al. Morphine amplifies mechanical allodynia via TLR4 in a rat model of spinal cord injury. Brain Behav Immun 2016;58:348-356.

40. Johnston IN, Milligan ED, Wieseler-Frank J, et al. A role for proinflammatory cytokines and fractalkine in analgesia, tolerance, and subsequent pain facilitation induced by chronic intrathecal morphine. J Neurosci 2004;24:7353-7365.

41. Agalave NM, Svensson CI. Extracellular high-mobility group box 1 protein (HMGB1) as a mediator of persistent pain. Mol Med 2015; 20: 569-578.

42. Frank MG, Weber MD, Fonken LK, Hershman SA, Watkins LR, Maier SF. The redox state of the alarmin HMGB1 is a pivotal factor in neuroinflammatory and microglial priming: A role for the NLRP3 inflammasome. Brain Behav Immun 2016;55:215-224.

43. Agalave NM, Larsson M, Abdelmoaty S, et al. Spinal HMGB1 induces TLR4-mediated long-lasting hypersensitivity and glial activation and regulates pain-like behavior in experimental arthritis. Pain 2014; 155:1802-1813.

44. Hutchinson MR, Zhang Y, Brown K, et al. Non-stereoselective reversal of neuropathic pain by naloxone and naltrexone: involvement of toll-like receptor 4 (TLR4). Eur J Neurosci 2008;28:20-29.

45. Li Y, Zhang H, Zhang H, Kosturakis AK, Jawad AB, Dougherty PM. Toll-like receptor 4 signaling contributes to Paclitaxel-induced peripheral neuropathy. J Pain 2014;15:712-725

46. Liu T, Han Q, Chen G, et al. Toll-like receptor 4 contributes to chronic itch, alloknesis, and spinal astrocyte activation in male mice. Pain 2016;157:806-817

47. Zhang Z, Liu Q, Liu M, et al. Upregulation of HMGB1-TLR4 inflammatory pathway in focal cortical dysplasia type II. J Neuroinflammation 2018;15:27-36.
48. Calvo-Rodríguez M, de la Fuente C, García-Durillo M, GarcíaRodríguez C, Villalobos C, Núñez. Aging and amyloid $\beta$ oligomers enhance TLR4 expression, LPS-induced $\mathrm{Ca}^{2+}$ responses, and neuron cell death in cultured rat hippocampal neurons. J Neuroinflammation. 2017;14:24-37.

49. Grace PM, Strand KA, Galer EL, Rice KC, Maier SF, Watkins LR. Protraction of neuropathic pain by morphine is mediated by spinal damage associated molecular patterns (DAMPs) in male rats. Brain Behav Immun 2018;72:45-50.

50. Eidson LN, Inoue K, Young LJ, et al. Toll-like Receptor 4 Mediates Morphine-Induced Neuroinflammation and Tolerance via Soluble Tumor Necrosis Factor Signaling. Neuropsychopharmacology 2017;42:661-670.

51. Franchi S, Moretti S, Castelli M, et al. Mu opioid receptor activation modulates Toll like receptor 4 in murine macrophages. Brain Behav Immun 2012;26:480-488.

52. Hutchinson MR, Lewis SS, Coats BD, et al. Possible involvement of toll-like receptor 4/myeloid differentiation factor-2 activity of opioid inactive isomers causes spinal proinflammation and related behavioral consequences. Neuroscience 2010;167:880-893.

53. Yang H, Hreggvidsdottir HS, Palmblad K, et al. A critical cysteine is required for HMGB1 binding to Toll-like receptor 4 and activation of macrophage cytokine release. Proc Natl Acad Sci U S A 2010;107:11942-11947.

54. Yang H, Lundback P, Ottosson L, et al. Redox modification of cysteine residues regulates the cytokine activity of high mobility group box-1 (HMGB1). Mol Med 2012;18:250-259.

Publisher's Note Springer Nature remains neutral with regard to jurisdictional claims in published maps and institutional affiliations. 